\title{
Article
}

\section{The nasal mutualist Dolosigranulum pigrum AMBR11 supports homeostasis via multiple mechanisms}

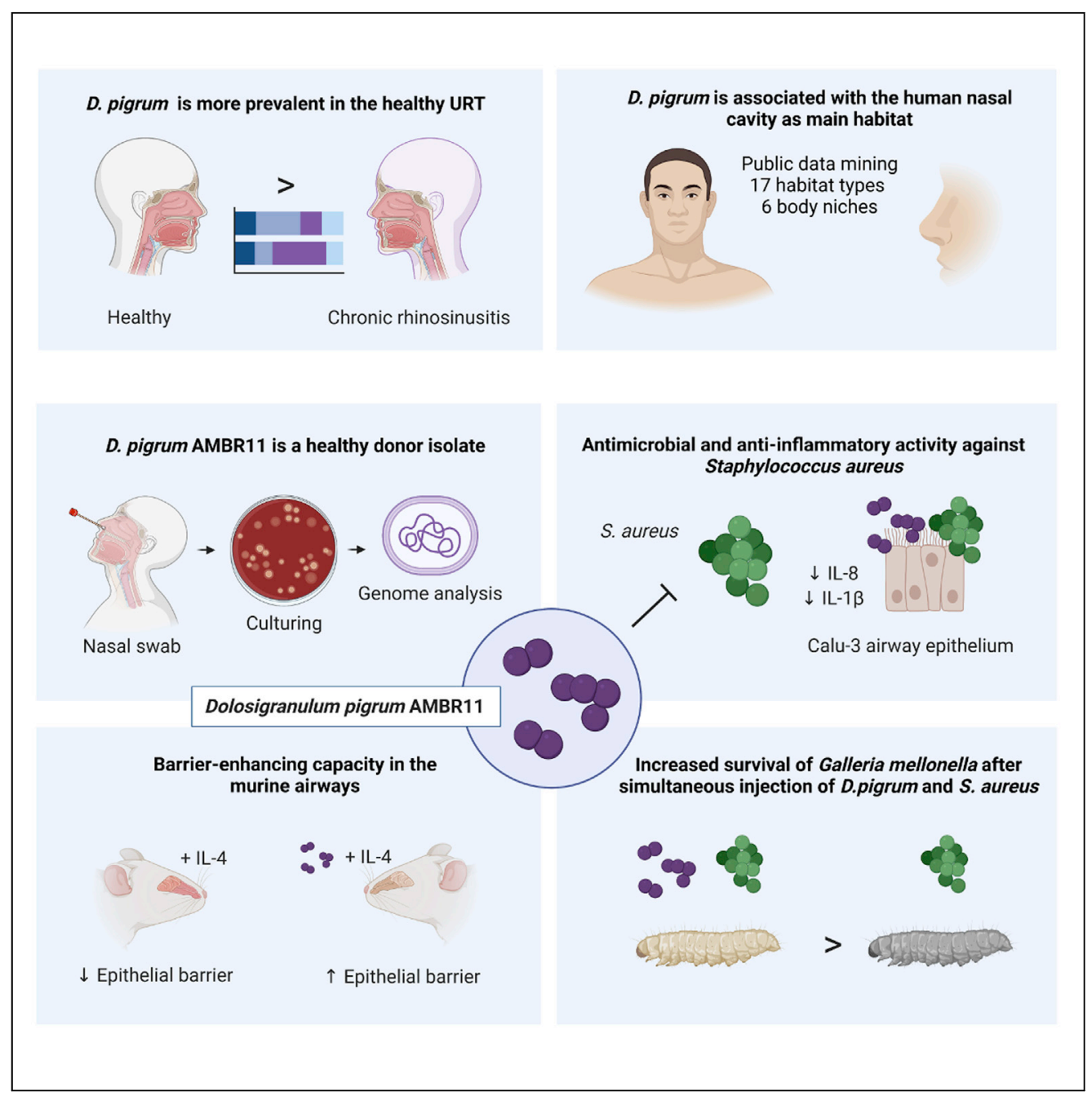

Ilke De Boeck, Stijn Wittouck, Katleen Martens, ..., Peter W. Hellings, Olivier M. Vanderveken, Sarah Lebeer

sarah.lebeer@uantwerpen.be

Highlights Habitat mining reveals that Dolosigranulum pigrum prefers the human nasal cavity

D. pigrum AMBR11 has immunomodulatory and nasal barrier-enhancing capacities

D. pigrum AMBR11 exerts antimicrobial activity against $S$. aureus 


\title{
Article \\ The nasal mutualist Dolosigranulum pigrum AMBR11 supports homeostasis via multiple mechanisms
}

\author{
Ilke De Boeck, ${ }^{1}$ Stijn Wittouck, ${ }^{1}$ Katleen Martens, ${ }^{1,2}$ Irina Spacova, ${ }^{1}$ Eline Cauwenberghs, \\ Camille Nina Allonsius, ${ }^{1}$ Jennifer Jörissen, ${ }^{1}$ Sander Wuyts, ${ }^{1}$ Wannes Van Beeck, ${ }^{1}$ Jelle Dillen, ${ }^{1}$ Peter A. Bron, \\ Brecht Steelant, ${ }^{2}$ Peter W. Hellings, ${ }^{2,3}$ Olivier M. Vanderveken, ${ }^{4,5}$ and Sarah Lebeer ${ }^{1,6, *}$
}

\begin{abstract}
SUMMARY
Comparing the nasal microbiome of healthy individuals and chronic rhinosinusitis (CRS) patients revealed Dolosigranulum pigrum as a species clearly associated with nasal health, although isolates obtained from healthy individuals are scarce. In this study, we explored the properties of this understudied lactic acid bacterium by integrating comparative genomics, habitat mining, cultivation, and functional characterization of interaction capacities. Mining 10.000 samples from the Earth Microbiome Project of 17 habitat types revealed that Dolosigranulum is mainly associated with the human nasal cavity. D. pigrum AMBR11 isolated from the nose of a healthy individual exerted antimicrobial activity against Staphylococcus aureus, decreased proinflammatory cytokine production in airway epithelial cells, and Galleria mellonella larvae mortality induced by this important nasal pathobiont. Furthermore, the strain protected the nasal barrier function in a mouse model using interleukin-4 as disruptive cytokine. Hence, D. pigrum AMBR11 is a mutualist with high potential as topical live biotherapeutic product.
\end{abstract}

\section{INTRODUCTION}

The microbiome of the upper respiratory tract (URT) has an important gatekeeper function by forming a barrier to potential pathogens and modulating immune responses (Man et al., 2017). The current knowledge on the URT microbiome is mainly based on association studies mapping the occurrence and relative abundance of different bacterial taxa by using culture-independent methods (Gan et al., 2021; Hasegawa et al., 2016; Lappan et al., 2018; Laufer et al., 2011). These studies have so far primarily revealed that many potential pathogens such as Streptococcus pneumoniae, Moraxella catarrhalis, Haemophilus influenzae, and Staphylococcus aureus are often present and prevalent in healthy subjects, implying that the host context and other resident microbiota must play a key role in many URT pathologies. Indeed, it becomes increasingly clear that disorders such as chronic rhinosinusitis (CRS) are not caused by an overgrowth of specific pathogens. For example, we could only link 1 to $2 \%$ of the variance in human nasal microbiome profiles to CRS versus health as a predictor in our previous microbiome comparison based on amplicon sequencing of the V4 region of the 16S rRNA gene (De Boeck et al., 2019). However, one of our most striking findings was the identification of potential beneficial taxa of the order Lactobacillales as being more prevalent and abundant in the healthy subjects compared to the CRS patients. This order of lactic acid bacteria (LAB) encompasses taxa from the family of the Lactobacillaceae, including well-characterized lactobacilli (De Boeck et al., 2020), and from the family of Carnobacteriaceae (Lawson and Caldwell, 2014), especially the species Dolosigranulum pigrum (De Boeck et al., 2019). Compared to (nasal) lactobacilli that could be cultured and studied in lab, animal and even in human trials (De Boeck et al., 2020; Martens et al., 2021; De Rudder et al., 2020), nasal Carnobacteriaceae turned out to be more difficult to culture and study at functional level. D. pigrum was only first described in 1993 as a Gram-positive, catalase-negative bacterium and potentially opportunistic pathogen (Aguirre et al., 1993). Since then, Dolosigranulum has been isolated and studied mainly in the context of disease (Laclaire and Facklam, 2000). However, their pathogenic nature is questionable and it is likely that most isolates were coincidently cultivated from some specific infection cases. In addition, several more recent URT comparative microbiome studies have identified this species as being a potentially beneficial taxon (Gan et al., 2019; Lappan et al., 2018). The study of Lappan and colleagues for instance found that the nasopharyngeal microbiome of healthy children had higher abundances of Dolosigranulum compared to children with recurrent acute otitis media (Lappan et al., 2018).

${ }^{1}$ Department of Bioscience Engineering, University of Antwerp,

Groenenborgerlaan 171, 2020 Antwerp, Belgium

${ }^{2} \mathrm{KU}$ Leuven Department of Microbiology, Immunology and Transplantation, Allergy and Clinical Immunology Research Unit, Herestraat 49, 3000 Leuven, Belgium

${ }^{3}$ Clinical Department of Otorhinolaryngology, Head and Neck Surgery, University Hospitals Leuven, Herestraat 49, 3000 Leuven, Belgium

${ }^{4}$ Faculty of Medicine and Health Sciences,

Translational Neurosciences, University of Antwerp, Universiteitsplein 1, 2610 Antwerp, Belgium

${ }^{5}$ ENT, Head and Neck Surgery and Communication Disorders, Antwerp University Hospital, 2650 Edegem, Belgium

¿Lead contact

*Correspondence: sarah.lebeer@uantwerpen. be

https://doi.org/10.1016/j.isci. 2021.102978 
Similar results were described in healthy adults compared to CRS in patients with nasal polyps (Gan et al., 2019). Nasal colonization with Dolosigranulum has also been associated with a decreased abundance of the important pathobiont S.aureus in adults (Brugger et al., 2020; Escapa et al., 2018; Yan et al., 2013) and in young children (Biesbroek et al., 2014a, 2014b). Unfortunately, most associative microbiota profiling studies lack subsequent functional characterization of individual species. A notable exception is a study by Brugger and colleagues, which reported on in vitro antimicrobial effects of $D$. pigrum strains against S. aureus (Brugger et al., 2020). Moreover, an inhibition of S. pneumoniae was also observed but this required a combination of $D$. pigrum and a nasal Corynebacterium. However, most D. pigrum strains used in this study were isolated in the context of disease or infection, and characterization was merely focused on its antimicrobial activity, so that the function and activity beyond its antimicrobial effects as beneficial mutualist remained to be substantiated. Indeed, for a microbe to be considered as a commensal or potential symbiont, its activities should go beyond mere effects on the microbial ecosystem, and direct beneficial effects such as barrier enhancing capacities or immunomodulatory role for the host should also be documented (Eloe-Fadrosh and Rasko, 2013).

In this study, a microbiome comparison of URT samples from 225 CRS patients and 100 healthy controls pointed at the genus Dolosigranulum as the taxon most clearly associated with health in terms of prevalence and abundance. Data mining of public microbiome data substantiated the human nose as the major habitat for this genus, of which D. pigrum is the only currently known species. Subsequently, to mechanistically validate the association of D. pigrum with a healthy URT detected at the microbiome level, we experimentally explored its anti-pathogenic, anti-inflammatory and barrier-promoting beneficial properties. Specific isolates were cultured from healthy noses of healthy individuals and assessed for anti-inflammatory characteristics in respiratory epithelial cells upon coculture with $S$. aureus, because this is one of the most important pathobionts in the human airways and associated with chronic airway disease (Derycke et al., 2010; Teufelberger et al., 2019). The interaction with the host was further substantiated in animal model studies, revealing that D. pigrum AMBR11 could decrease mortality of Galleria mellonella larvae after S. aureus injection and reduce IL-4-induced nasal barrier dysfunction in mice.

\section{RESULTS}

\section{Dolosigranulum pigrum is metabolically active and more prevalent in the healthy URT}

We recently established association between URT health and lactobacilli (De Boeck et al., 2020). This triggered us to revisit our observational microbiome study of healthy controls versus CRS patients (De Boeck et al., 2019) with full focus on taxa belonging to the Carnobacteriaceae, because they were even more strongly linked to health based on a compositional differential abundance analysis (De Boeck et al., 2020), but not yet explored at functional level. Only one abundant genus of Carnobacteriaceae was detected, namely Dolosigranulum. The genus Dolosigranulum was detected in the anterior nares of $81 \%$ versus only $54 \%$ in CRS patients $(p<0.0001)$. For the nasopharynx, the prevalence of this genus was $78 \%$ in healthy adults versus $42 \%$ of the CRS patients $(p<0.0001)$ (Figure 1B). The median relative abundance of Dolosigranulum in the anterior nares was also 3 -fold higher in the control group compared to the CRS group (Figure 1C). In the nasopharynx, no significant difference in fold change was observed. As DNA-based technologies, such as the above, cannot discriminate between live and dead bacteria, RNA-based 16S rRNA gene sequencing was also performed on a subset of healthy control samples (Figure 1A). The RNA approach was able to detect this D. pigrum ASV with similar relative abundances $(30 \%$ and $37 \%$ for DNA versus RNA sequencing in anterior nares, and $19 \%$ versus $15 \%$ for DNA versus RNA in nasopharynx samples, respectively) (Figure 1D), indicating that D. pigrum is not only more abundant in healthy individuals than in CRS patients, but also an active member of the healthy URT bacterial community.

\section{Comparative genomics of healthy donor-isolated Dolosigranulum pigrum}

To isolate D. pigrum strains for subsequent functional analyses on potential beneficial properties, samples derived from healthy nasal swabs were cultivated. After 2-3 days of growth, single colonies were used for full length 16S rRNA gene based identification, revealing that larger colonies, typically representing faster growing strains, mainly belonged to Staphylococcus, Streptococcus, and Corynebacterium species. By contrast, two of the smaller-sized colonies were identified as D. pigrum, in line with earlier observations that members of the Carnobacteriaceae display slow growth under laboratory conditions (Afzal et al., 2010). These isolates were designated as Dolosigranulum pigrum AMBR11 and AMBR12 and deposited in the Belgian Culture collection of Microbes (LMG P-31124 and LMG P-31154, respectively). Subsequently, 

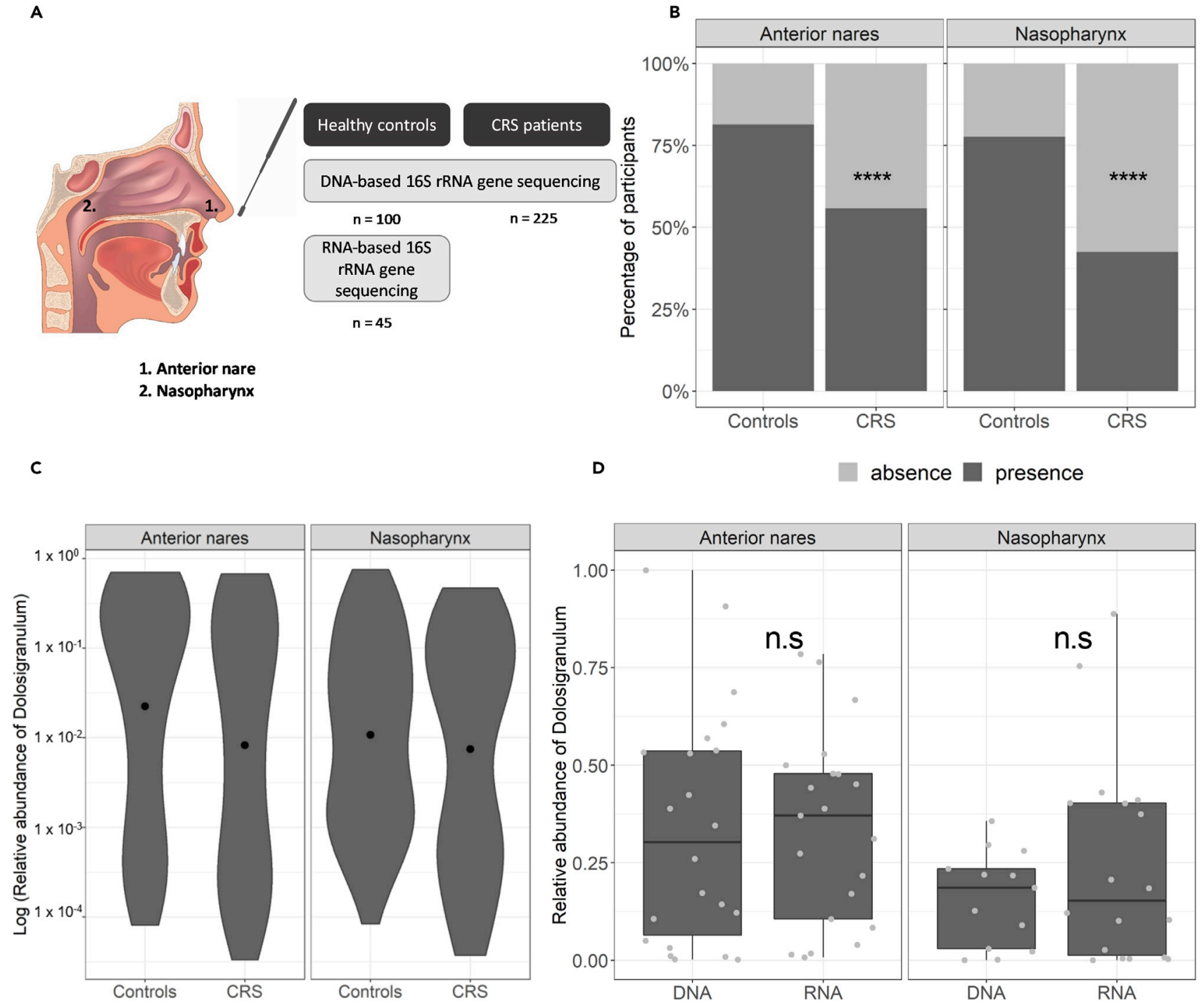

Figure 1. Dolosigranulum in the anterior nares and nasopharynx of 100 healthy participants and 225 CRS patients

(A) Overview of the study groups and collected samples for both sequencing approaches.

(B and C) 16S V4 rRNA URT microbiome sequencing analyzed for the prevalence (B) and relative abundance (C) of the Dolosigranulum ASV in 100 healthy controls versus 225 CRS patients. The means per location and condition are indicated with a black dot. ${ }^{\star * * \star} p<0.0001$ (Fisher exact test).

(D) Relative abundance of Dolosigranulum in a subset of anterior nares and nasopharynx samples ( $n=45)$ with DNA versus RNA-based $16 S$ rRNA sequencing. $p$ values for comparison of mean relative abundance between DNA versus RNA sequencing were determined using Wilcoxon rank-sum test; $\mathrm{n}$.s. = not significant.

total DNA of pure cultures was extracted and subjected to whole genome sequencing. D. pigrum AMBR11 and AMBR12 were then revealed to harbor genome sizes of 1.88 and $1.90 \mathrm{Mb}$ with $\mathrm{GC}$ contents of $39.6 \%$ and $39.7 \%$, respectively. These genome features are highly comparable to what was observed earlier for related strains isolated from a disease context (Brugger et al., 2020), where an average genome size of $1.86 \mathrm{Mb}$ was established for the previous 11 strains evaluated. The genome sizes for Lactobacillales species with a postulated free-living (e.g. Lentilactobacillus kefiri, $2.4 \mathrm{Mb}$ ) or nomadic (e.g. Lacticaseibacillus rhamnosus, $2.8 \mathrm{Mb}$ ) lifestyle are typically larger (Figure S1) (Duar et al., 2017). This relatively small genome size suggests a host-adapted lifestyle, in line with other host-adapted members of the lactic acid bacteria, such as Lactobacillus gasseri (1.8 Mb). Pairwise-genome comparison revealed average nucleotide identity (ANI) values of $97.7 \%$ for both strains with the type strain D. pigrum LMG15126, confirming that the isolated strains are appropriately classified as the species $D$. pigrum. 


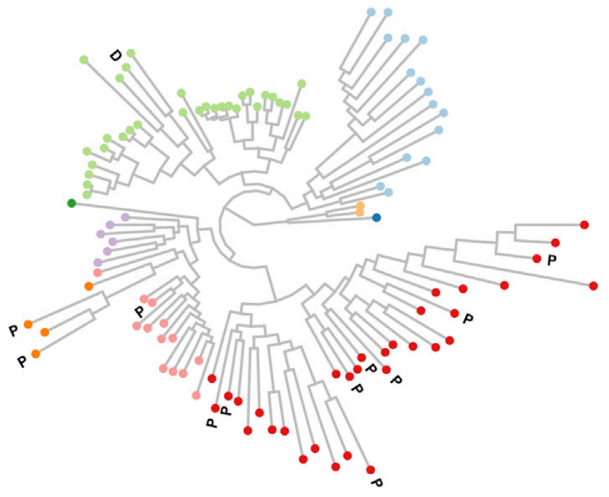

B

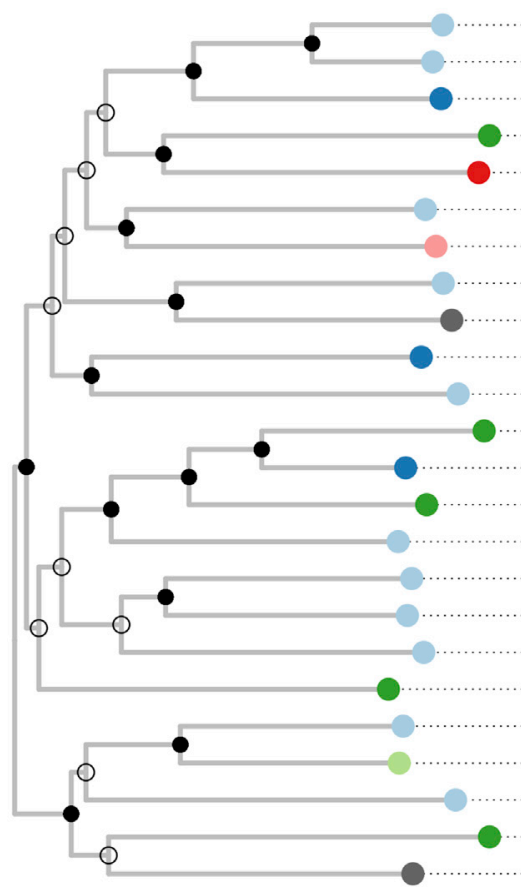

family

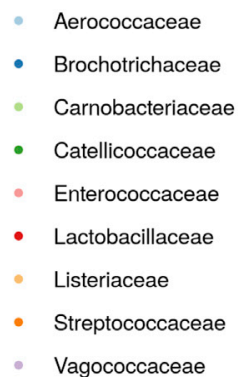

- Aerococcaceae

- Brochotrichaceae

- Carnobacteriaceae

- Catellicoccaceae

- Enterococcaceae

Streptococcaceae

- Vagococcaceae

\begin{tabular}{|c|c|c|}
\hline $\begin{array}{l}\text { KPL1914 } \\
3300007883\end{array}$ & & \\
\hline KPL1931 & & \\
\hline KPL1939 & & \\
\hline ATCC 51524 & & \\
\hline $3300007943 \_5$ & & \\
\hline KPL1922 & & \\
\hline $\begin{array}{l}\text { 3300007745_2 } \\
15 S 00348\end{array}$ & body & y site \\
\hline $\begin{array}{l}15 S 00348 \\
\text { KPL1937 }\end{array}$ & - & anterior nares \\
\hline 3300008349_3 & & blood \\
\hline KPL1938 & - & eye \\
\hline KPL1932 & - & nasopharynx \\
\hline AMBR11 * & 0 & sinus \\
\hline 3300007942_2 & - & spinal cord \\
\hline 3300007365_2 & & NA \\
\hline 3300006993_2 & & \\
\hline $3300006676 \_5$ & & \\
\hline KPL1930 & & \\
\hline 3300007936_1 & & \\
\hline KPL1934 & & \\
\hline AMBR12 * & & \\
\hline KPL1933 & & \\
\hline 83VPs-KB5 & & \\
\hline
\end{tabular}

Figure 2. Comparative genomics of $D$. pigrum

(A) Maximum likelihood phylogenetic tree of Lactobacillales inferred on a concatenated alignment of the amino acid sequences of 389 (almost) single-copy core genes. One representative genome per species of Carnobacteriaceae was used, and one genome per genus of non-Carnobacteriaceae. $\mathrm{D}=$ Dolosigranulum pigrum. $\mathrm{P}=$ genera that contain documented probiotic strains from Lactobacillus, Limosilactobacillus, Lacticaseibacillus, Ligilactobacillus, Lactiplantibacillus, Latilactobacillus, Streptococcus, Enterococcus, Lactococcus, Leuconostoc and Pediococcus. (B) Maximum likelihood phylogenetic tree of Dolosigranulum pigrum strains inferred on a concatenated alignment of the nucleotide sequences of the top 1,000 most single-copy core genes. Branches with a SH-aLRT (Shimodaira-Hasegawa approximate likelihood ratio test) support value of $\geq 80 \%$ and bootstrap support of $\geq 95 \%$ were considered trustworthy and were marked with a filled dot; other branches were marked with an open dot. Healthy isolates are written in bold, other strains were isolated in the context of disease. The two $D$. pigrum strains isolated in this study are indicated with an asterisk. See also Figures S1 and S2.

Subsequently, we employed a phylogenomics approach to assess potential clustering of D. pigrum strains by body site or disease status, as well as the position of the species within the Lactobacillales. This order contains many taxa associated with the human host or with beneficial properties as probiotics (Zheng et al., 2020), but also pathogenic taxa such as Streptococcus pneumoniae (Du Toit et al., 2014). Initially, a phylogenetic tree of the order Lactobacillales was made, confirming that Dolosigranulum is an integral member of the Lactobacillales (Figure 2A). However, within this order, D. pigrum is only distantly related to welldocumented beneficial genera for human health, such as the Lactobacillus crispatus, Lactobacillus gasseri, 
Lactobacillus jensenii, and Lactobacillus iners species that are linked to vaginal health (Petrova et al., 2015), Limosilactobacillus reuteri and Ligilactobacillus ruminis linked to gastro-intestinal health of humans and other vertebrates as gut symbionts (Oh et al., 2010), and Lacticaseibacillus as one of the most widely used probiotic genera for human and even other animal applications (Zheng et al., 2020) to which also our previously documented nasal probiotic strain L. casei AMBR2 belongs (De Boeck et al., 2020). The closest known relative of $D$. pigrum in this comparison was Alloiococcus otitis, a species typically isolated from URT, more specifically from middle-ear fluid in children with otitis media with effusion, where it is a possible pathobiont (Faden and Dryja, 1989).

Intraspecies comparison by a phylogenetic tree containing 24 D. pigrum genomes, including genomes of our own 2 isolates, 9 metagenome assembled genomes (MAGs) from the Genomes from Earth's Microbiomes (GEM) catalog (Nayfach et al., 2020), and 13 publicly available genomes from isolates (11 of which were previously evaluated (Brugger et al., 2020)), revealed that strains isolated from the same body site did not cluster together (Figure 2B). We found that D. pigrum has 1,284 core genes, whereas the average number of accessory genes in the full set of genomes was 461. D. pigrum AMBR12 represents a strain with a close-to-average number of accessory genes (453), whereas AMBR11 harbors a relatively high number of 653 (Figure S2). Of these accessory genes, 242 and 12 genes were unique for strain AMBR11 and AMBR12, respectively. Notably, the 9 D. pigrum MAGs were all reconstructed from (apparently healthy) nare samples.

As a genomic assessment of the commensal nature of our D. pigrum AMBR11 and AMBR12 strains, the corresponding genomes were also screened using the Virulence Factors Database (VFDB) (Liu et al., 2019), a validated and frequently used online database that provides up-to-date knowledge of virulence factors from common bacterial pathogens. No positive hits were detected when the genomes were screened against the core nucleotide database, which consists of experimentally validated virulence factors, indicating that $D$. pigrum does not encode any genes closely related to known virulence factors. Similarly, no match with known antibiotic resistance genes was detected for AMBR11 and AMBR12.

\section{Habitat mining of Dolosigranulum pigrum reveals a preference for the human nasal cavity}

We next aimed to explore the habitat range of Dolosigranulum. Because the genus was so distinctly detected in our samples of the anterior nares and nasopharynx (Figure 1), we first explored its presence and relative abundance in other habitats of the human body based on publicly available shotgun sequencing data (Pasolli et al., 2017). In total, 8,184 samples from six different body sites were analyzed, i.e., nasal cavity $(n=93)$, oral cavity $(n=701)$, skin $(n=512)$, stool $(n=6,784)$, vagina $(n=86)$, and breastmilk $(n=8)$. Dolosigranulum was detected in 38 nasal cavity samples $(41 \%), 75$ skin samples (15\%), one milk sample (12.5\%), 36 stool samples (0.53\%), one oral cavity sample (0.14\%), and not in vaginal samples (Figure $3 \mathrm{~A}$ ). The relative abundance of Dolosigranulum in the nasal cavity was $18 \%$ on average, far exceeding the relative abundances found for the other human body sites, e.g. on average 1.28\% for skin samples, which had the second highest relative abundances (Figure 3B), reiterating our 16S-based analysis of nasopharynx and anterior nare samples of healthy subjects and CRS patients described above (Figure 1).

Besides the human body, we explored other potential habitats of D. pigrum. Its prevalence and relative abundance was mined in 17 habitat types including free-living environments (e.g. water, sediment, and soil) and host-associated environments ( 3 different plant and 5 different animal environments) using publicly available microbiome data from the Earth Microbiome Project (EMP), where the largest subset of 10,000 samples was used (Thompson et al., 2017). Dolosigranulum was generally not detected in the environmental samples analyzed (including water, soil, and plant samples), but was present (defined as $>1$ reads per 10,000 reads or $0.01 \%$ relative abundance) in animal samples (Figure 3 C). We therefore further evaluated its prevalence in 43 animal host species based on the EMP dataset, including rodents (Rattus norvegicus), fish (Ictalurus punctatus, Cyprinus carpio, Catostomus), birds (Gila robusta), and primates (Pygathrix nemaeus, Nasalis larvatus), but its relative abundance was $<1 \%$ (Figure 3D). In contrast, in humans and dogs (Canis lupus familiaris), Dolosigranulum was found in high relative abundances in some samples, up to almost 50\% (Figure 3D). Interestingly, the samples from dogs with high relative abundances were all annotated as nasal secretions (Gilbert et al., 2014). Thus, our extensive data mining of more than 10,000 publicly available microbiome samples revealed that Dolosigranulum is almost exclusively found in the nasal cavity. Considering its relatively small genome size and its clear association with the human nose as habitat, it is likely that this species has a specific function in this ecosystem. 
A

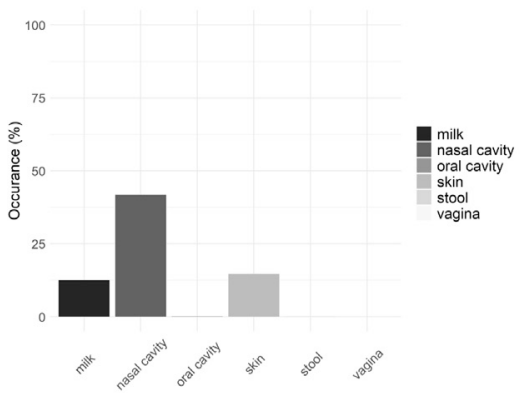

C

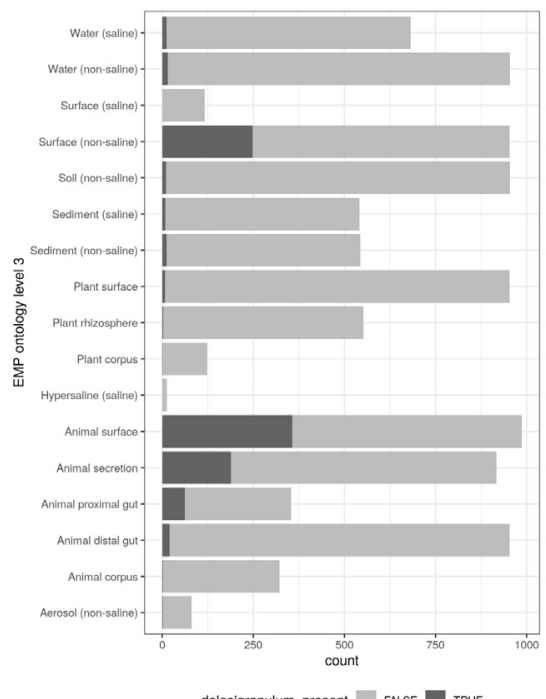

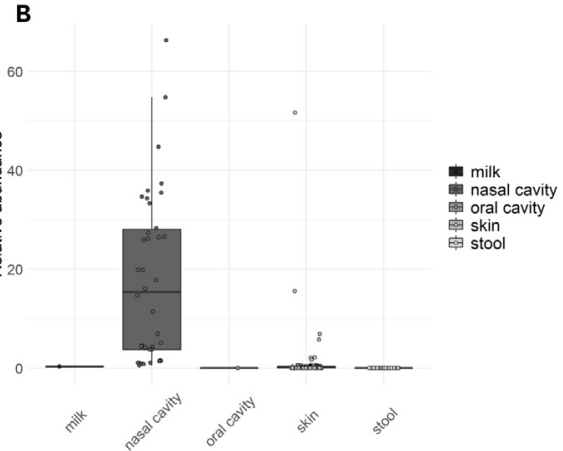

D

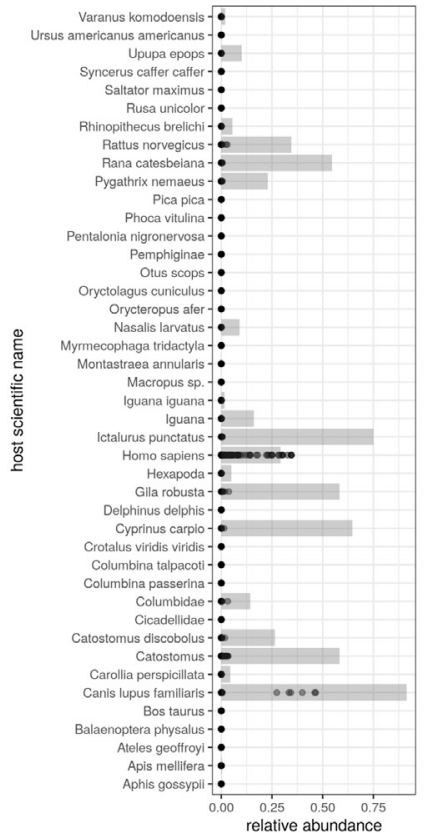

Figure 3. Presence of Dolosigranulum sequences in different environmental samples and host species, and within different body sites of the human host based on public microbiome data mining

(A) Percentage of Dolosigranulum in samples from human milk, nasal cavity, oral cavity, skin, stool, and vagina of the shotgun metagenomics datasets, accessed through the curated Metagenomics R package.

(B) Comparison of relative abundance of Dolosigranulum in the same human body site. Vaginal samples are not shown, as no Dolosigranulum was detected in these samples.

(C) Occurrence of Dolosigranulum in 17 different habitats including environmental, plant and animal samples, using the EMP dataset (subset of 10,000 samples) (Thompson et al., 2017). Species with less than 10 samples in the EMP dataset are not depicted.

(D) Prevalence and relative abundance of Dolosigranulum in different animal host species, using the EMP dataset. Grey bars represent the prevalence, while dots represent the relative abundances in individual samples. Only species with ten or more samples available are depicted.

\section{Antimicrobial properties of D. pigrum AMBR11 against S. aureus}

As a first function screened for, we functionally assessed the potential antimicrobial effects of our healthy isolate D. pigrum AMBR11 against Staphylococcus aureus, a major URT pathobiont in CRS and also in other chronic airway diseases (Martina et al., 2019; Teufelberger et al., 2019). The AMBR11 isolate was selected for further functional analysis because this strain displayed better growth characteristics than isolate AMBR12 and showed more unique accessory genes (242 compared to 12 for AMBR12). The antimicrobial activity of D. pigrum AMBR11 was profiled and benchmarked against the D. pigrum type strain LMG15126 (isolated from a human spinal cord autopsy) and against the established effects of model probiotic lactic acid bacteria, namely, the commercial gut probiotic strain L. rhamnosus GG (van den Broek et al., 2018) and 


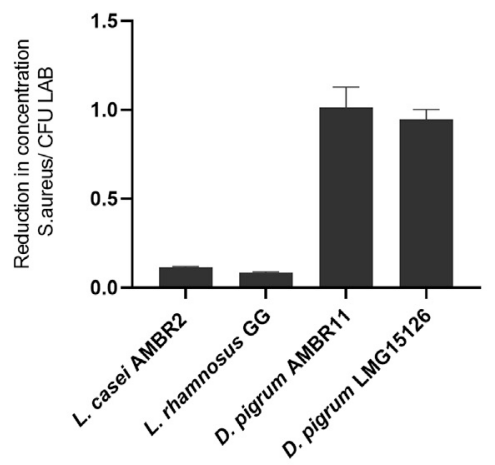

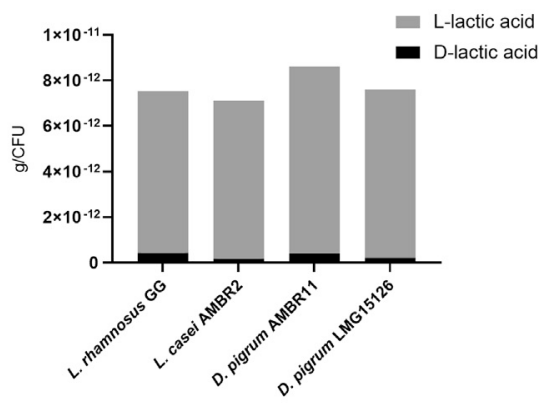

C

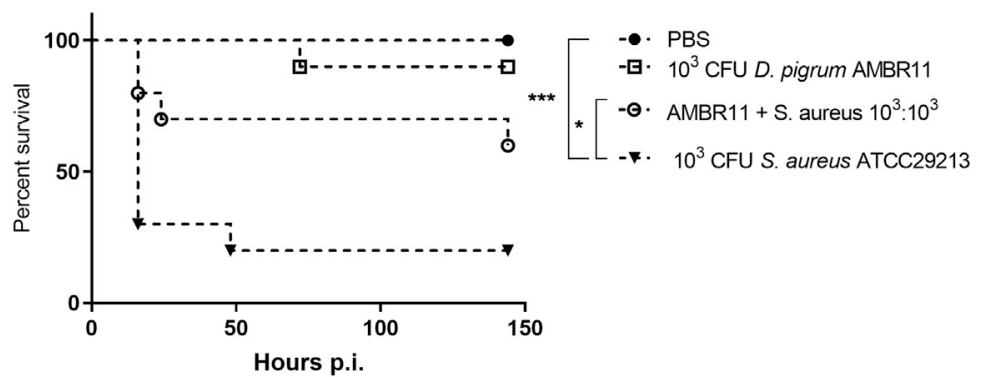

Figure 4. Antimicrobial effects of $D$. pigrum AMBR11 against S. aureus

(A) Antimicrobial effects of CFS of D. pigrum AMBR11, D. pigrum LMG15126, L. rhamnosus GG and L. casei AMBR2, against S. aureus ATCC29213 were evaluated after $28 \mathrm{~h}$. Results were normalized to the number of LAB producer cells (CFU/mL). After 16 h of growth in $\mathrm{BHI}$ with $0.05 \%$ Tween medium, Dolosigranulum reached a final concentration of $5 \times 10^{7}$ $\mathrm{CFU} / \mathrm{mL}$ while the lactobacilli tested $10^{9} \mathrm{CFU} / \mathrm{mL}$ in MRS medium. Data are presented as means \pm standard deviations. (B) L- and D-lactic acid production by L. rhamnosus GG, L. casei AMBR2, D. pigrum LMG15126, and D. pigrum AMBR11 after overnight incubation. Results are expressed as $\mathrm{g} / \mathrm{CFU}$.

(C) Cocultures of D. pigrum AMBR11 $\left(10^{3} \mathrm{CFU} / \mathrm{mL}\right)$ with $\mathrm{S}$. aureus ATCC29213 $\left(10^{3} \mathrm{CFU} / \mathrm{mL}\right)$ were inoculated in the haemolymph of the larvae and survival was studied for 144 hours post-infection (p.i.). Results were compared with monocultures of $S$. aureus. Pairwise comparisons of survival curves of treated groups with the survival curve of the control PBS condition, or between the S. aureus and AMBR11 + S. aureus group, were performed in GraphPad Prism 8.4.3 using the de Log rank (Mantel-Cox) test. Where applicable, this test was preceded by the Bonferroni correction for multiple comparisons. ${ }^{* *} \mathrm{p}<0.001$ and ${ }^{*} \mathrm{p}<0.05$ between indicated groups.

the URT-adapted probiotic candidate L. casei AMBR2 (De Boeck et al., 2020). The two Lacticaseibacillus and two D. pigrum strains tested were able to partially inhibit the growth of S. aureus ATCC29213. $D$. pigrum displayed the highest activity when expressed per colony-forming unit (CFU) (Figure 4A). Because lactic acid is a hallmark metabolite of lactic acid bacteria (De Keersmaecker et al., 2006), lactic acid production of $D$. pigrum AMBR11 and the type strain were also compared to $L$. rhamnosus GG and L. casei AMBR2 after overnight incubation (Figure 4B). In agreement with their classification as LAB within the order Lactobacillales (Figure 2B), D. pigrum AMBR11 and LMG15126 cells produced comparable L-lactic acid levels per CFU to the lactobacilli tested, while only low levels of D-lactic acid were found. This suggests that L-lactic acid and the consequential local low-pH environment could be one of the drivers of the observed S. aureus inhibition by AMBR11, while at the same time it is likely that other, currently unknown factors might explain the superior inhibitory effect observed for D. pigrum.

With the established S. aureus growth-inhibition properties of strain AMBR11 in vitro, we set out to investigate the actual capacity of our strain to reduce $S$. aureus infection in an animal model employing Galleria mellonella. In this model, the survival percentage of larvae was investigated when D. pigrum AMBR11 and S. aureus ATCC29213 were injected individually, as well as in coculture (Figure 4C). Negative controls (PBS or D. pigrum injection) resulted in high survival rates of the larvae (up to 100\%). By contrast, S. aureus 
A

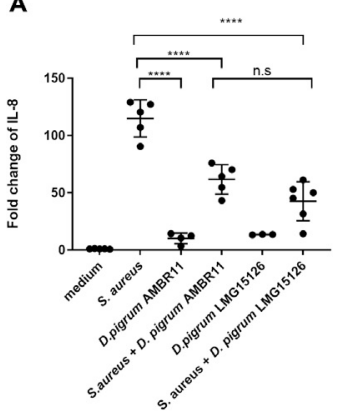

D

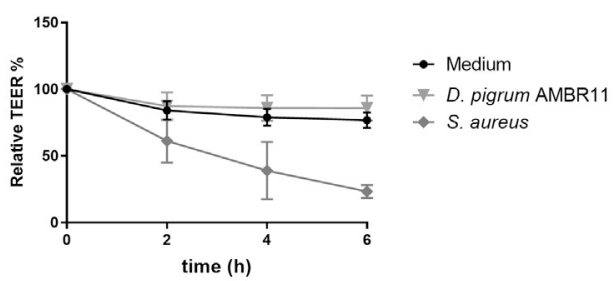

$\mathbf{F}$

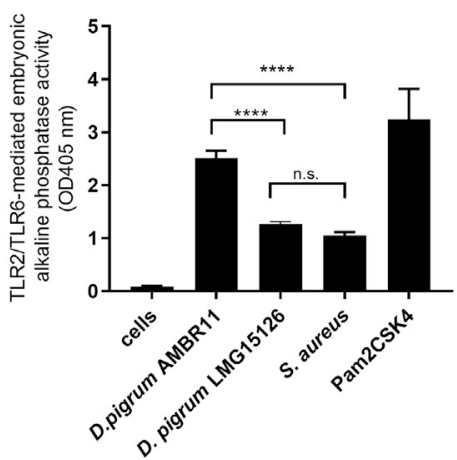

B

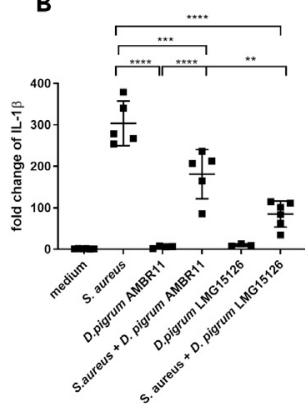

C

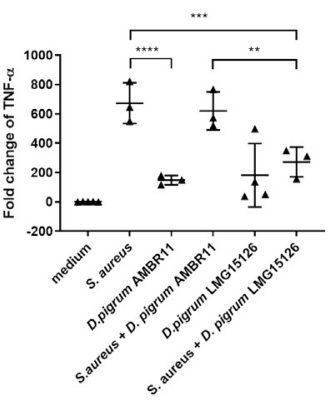

G

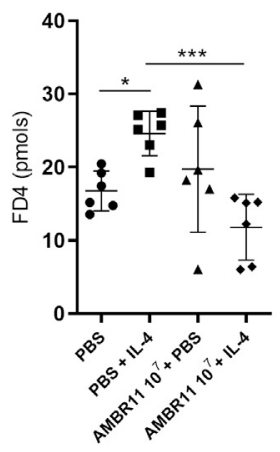

Figure 5. The effect of $D$. pigrum AMBR11 on the airway epithelial barrier in vitro and in vivo

(A-C) Induction of IL-8 (A), IL-1 $\beta$ (B), and TNF- $\alpha$ (C) mRNA by S. aureus as such or upon co-incubation with URT isolate D. pigrum AMBR11 or type strain D. pigrum LMG15126 with the Calu-3 cells. Bacteria were incubated with the cells for 4 hours in a final concentration of $10^{8} \mathrm{CFU} / \mathrm{mL}$. The presented data are representative of two independent experiments. Data are expressed as means \pm standard deviations. Significant differences between all tested conditions were evaluated with one-way ANOVA and multiple t tests in GraphPad Prism. The p value was corrected for multiple comparisons using the Holm-Sidak's multiple comparisons test. Significant differences compared to $S$. aureus are indicated with an asterisks: ${ }^{* *} p<0.01,{ }^{* \star *} p<0.001,{ }^{* \star * *} p<0.0001$. Significant differences between all tested conditions are shown in Table S1.

(D) Evaluation of TEER to measure epithelial barrier integrity for 6 hours after addition of D. pigrum AMBR11 and S. aureus on primary NECs from CRSwNP patients $(n=4)$. TEER values are expressed as relative percentages against the initial TEER. Bacterial strains were added in a concentration of $10^{7} \mathrm{CFU} / \mathrm{mL}$ and TEER was measured every 2 hours. Cells as such were used as negative control. Results are expressed as the mean with standard deviation.

(E) Murine model of IL-4-induced barrier disruption. Mice were endonasally pre-treated two times with D. pigrum AMBR11 (107 CFU/ml). 24 hours later, IL-4 was applied three times with one hour interval. 24 hours after the last IL-4 application, FD4 was applied endonasally to evaluate mucosal permeability in the serum of the treated mice. Data are presented as mean \pm standard deviation. The Mann Whitney test in Graphpad prism was used for statistical differences. 
Figure 5. Continued

(F) Activation of TLR2/TLR6 by D. pigrum AMBR11, D. pigrum LMG15126 and S. aureus. Pam2CKS4 was used as positive control. Significant differences between the bacterial conditions are indicated with an asterisks. ${ }^{\star \star \star \star} p<0.0001$. Data are presented as mean \pm standard deviation

(G) Activation of TLR2/TLR1 by D. pigrum AMBR11, D. pigrum LMG15126 and S. aureus. Pam3CKS4 was used as positive control. Significant differences between the bacterial conditions are indicated with an asterisks. ${ }^{\star} p<0.05,{ }^{\star \star * \star} p<0.0001$. Data are presented as mean \pm standard deviation.

infection resulted in death of the vast majority of the larvae. The survival percentages of larvae exposed to $S$. aureus in combination with $D$. pigrum AMBR11 concentration was substantially improved $(p<0.05)$, demonstrating its in vivo protective effect.

\section{Anti-inflammatory and barrier-enhancing capacity of D. pigrum AMBR11}

Since microbe-microbe effects are insufficient to characterize the mutualistic nature of a microbe, we also studied phenotypes reflecting microbe-host interactions. First, we assessed whether D. pigrum AMBR11, besides the direct antimicrobial activity established above, could also reduce the typical pro-inflammatory cytokine profile induced by S. aureus ATCC29213 (Szkaradkiewicz et al., 2012). Possible anti-inflammatory capacities of D. pigrum AMBR11 and type strain D. pigrum LMG15126 were evaluated by assessing their capacity to reduce inflammation induced by S. aureus ATCC29213 in Calu-3 respiratory epithelial cells. This inflammation was reflected by a significantly higher expression of mRNA for the pro-inflammatory markers interleukin (IL)-8, IL-1 $\beta$, and TNF- $\alpha$ in Calu-3 cells stimulated with S. aureus ATCC29213 compared to D. pigrum AMBR11 and D. pigrum LMG15126 (Figures 5A-5C). Importantly, the expression of both interleukin (IL)-8 and IL-1 $\beta$ was significantly decreased when S. aureus ATCC29213 was co-incubated with D. pigrum AMBR11 or LMG15126, showing that D. pigrum AMBR11 and LMG15126 exerted dampening effects on the inflammation induced by $S$. aureus.

Subsequently, we studied the host interaction capacity of $D$. pigrum with the nasal barrier, because restoration of the epithelial barrier can be considered a beneficial homeostasis-promoting property for a nasal probiotic or symbiont (Hellings and Steelant, 2020). Pathogens such as S. aureus are known to disrupt the nasal epithelial barrier, especially via their enterotoxins (Martens et al., 2021) but also other mechanisms have been described - for instance, via serine protease-like proteins (Chandrabalan et al., 2021). Here, we confirmed that administration of live S. aureus on primary NECs from CRS donors was able to disturb the nasal epithelial barrier, reflected via a decrease in relative TEER; however, live D. pigrum AMBR11 did not affect the barrier (Figure 5D). Barrier disruption is also observed independent of S. aureus infection for CRS and also for other URT diseases, such as asthma (Xiao et al., 2011) and allergic rhinitis (Steelant et al., 2016), as a side effect of air pollution (Zhao et al., 2018). Here, we evaluated the barrier-enhancing capacity of $D$. pigrum in a murine model of IL-4-induced nasal barrier dysfunction (Steelant et al., 2018). Mice were pre-treated twice endonasally with D. pigrum AMBR11 prior to IL-4 application, an agent known to disrupt the epithelial barrier (Steelant et al., 2016). This is reflected by a significant increase in fluorescein isothiocyanate-dextran (FD4) passage for the positive control $(\mathrm{PBS}+\mathrm{IL}-4)$. Pre-treatment with D. pigrum AMBR11 prevented this IL-4-induced increase in FD4 passage (Figure 5E, $\mathrm{P}<0.001$ ). D. pigrum AMBR11 without prior IL-4 treatment did not show significant effects on FD4 passage.

Finally, we explored the activation of some important Toll-like receptors (TLR) involved in epithelial barrier functioning for $D$. pigrum AMBR11. The results were compared against the $D$. pigrum type strain and URT pathobiont $S$. aureus. D. pigrum AMBR11 mainly activated the TLR2/TLR6 heterodimer at a significantly higher level ( $p<0.0001$ ) compared to $S$. aureus (Figure 5F). In contrast, stimulation of TLR2/TLR1 by D. pigrum was significantly lower compared to the pathobiont S. aureus (Figure 5G), showing that D. pigrum rather exploits TLR2/6 interaction mechanisms with host cells.

\section{DISCUSSION}

Lactic acid bacteria are among the most studied probiotic bacteria worldwide, mostly for gut and urogenital health. D. pigrum is an underexplored lactic acid bacterium, with the notable exception of some recent microbiome sequencing studies in which this species was associated with healthy airway conditions (Biesbroek et al., 2014b; De Boeck et al., 2019; Bosch et al., 2016; Brugger et al., 2020; Gan et al., 2021). Here, we 
gained fundamental insights into the lifestyle and beneficial host interaction capacity of Dolosigranulum with a combination of isolation of strains from healthy subjects, comparative genomics, microbiome habitat mining, and functional characterization of antimicrobial and host interactions.

Despite its high abundance in the URT at DNA and RNA level, Dolosigranulum cultivation and isolation turned to be more complex than initially anticipated because of frequent cross-contamination with fastgrowing species such as $S$. aureus and - in hindsight - also due to its narrow host and habitat range. The latter is also reflected in the small genome sizes of $1.88 \mathrm{Mb}$ and $1.9 \mathrm{Mb}$ of the URT-isolated D. pigrum AMBR11 and AMBR12, isolated in this study, and is in agreement with other D. pigrum genome sizes (Brugger et al., 2020). This small genome size is related to the postulated host-adapted features, as is typical for commensals and symbionts that often have undergone and are still undergoing evolutionary events that lead to genomic decay and loss of functions that are redundant in the host (Kirchberger et al., 2020). Also in the Lactobacillaceae, a reduced genome size is strongly correlated with obligate host-adaptation (Duar et al., 2017). For instance, the genome of L. iners is only $1.27 \mathrm{Mb}$, reflecting strong adaptation to the human vagina (Macklaim et al., 2011). In addition to a smaller genome size, a reduction in GC content has been associated with host-adaptation in lactobacilli; for instance, 32.5\% (L. iners), 38.6\% (L. reuteri), and 43.5\% (Ligilactobacillus ruminus) (Duar et al., 2017). Here, a GC content of 39.6\% was observed in D. pigrum AMBR11. As additional level of our ecological and functional characterization, we mined public data of $D$. pigrum in 17 different habitats including more than 10,000 environmental, plant, and animal samples, revealing that $D$. pigrum is highly associated with animal samples and mainly with the human host. Further exploration of this host-adapted nature pointed at an association with the human respiratory tract and nasal cavity. Taken together, our microbiome, genomic, and functional data suggest that Dolosigranulum has a mutualistic role in the human nose, justifying further functional research into its role as a beneficial member of the URT.

A first main function of Dolosigranulum appears to be inhibiting overgrowth of pathogens. This has already been previously suggested by Brugger et al.(Brugger et al., 2020), but was further refined here in an in vivo model for our isolate D. pigrum AMBR11 from a healthy donor. This health-associated strain showed a clear inhibitory effect on the growth of $S$. aureus based on microbe-microbe assays and in the invertebrate Galleria model, because the virulent effects of $S$. aureus in the larvae was dampened upon co-administration of $D$. pigrum AMBR11. This antimicrobial effect is likely related to its lactic acid production, which was in the range of other members of the Lactobacillaceae (van den Broek et al., 2018). Previous research has shown that lactic acid is also able to inhibit the growth of other important URT pathobionts, such as Moraxella catarrhalis (van den Broek et al., 2018) and other Gram-negatives (Alakomi et al., 2000). However, other antimicrobial compounds such as bacteriocins (Perez et al., 2014) cannot be ruled out at this stage, as already suggested based on previous analysis on D. pigrum strains (Brugger et al., 2020). To distinguish the activity of lactic acid in D. pigrum AMBR11 compared to bacteriocins or other antimicrobials, the purification and further identification of such molecules is of interest for future research, for instance, via mass spectrometry methods. Pathogen-inhibiting characteristics for D. pigrum strains studied from more disease context were also described by Brugger and colleagues (Brugger et al., 2020) and are in line with what was observed here. Furthermore, the same research group also performed a recent species-level reanalysis of a pediatric nostril microbiota dataset and adult nostril samples and found a negative association between relative abundances of $S$. aureus and D. pigrum based on an analysis of composition of microbiomes of microbiome data from pediatric nostril samples.

As another function in addition to these previously suggested antimicrobial effects, we observed anti-inflammatory effects important for D. pigrum potentially to maintain homeostasis. Indeed, the inflammatory response against $S$. aureus in the airway epithelial Calu-3 cells was diminished upon coculture with our strain D. pigrum AMBR11. This mechanism is in line with the fact that $D$. pigrum was previously associated with URT health and lower inflammation grades based on microbiome studies (Biesbroek et al., 2014b; Gan et al., 2021; Lappan et al., 2018). For example, in young children, D. pigrum was found to be significantly higher in the control group compared to children prone to recurrent acute otitis media based on $16 \mathrm{~S}$ rRNA gene sequencing (Lappan et al., 2018). In adults, a depletion of Dolosigranulum was found in the middle meatus of CRS patients with nasal polyposis compared to a control group (Gan et al., 2019). In these patients, an important role for $S$. aureus and its enterotoxins was established in the disease pathology and associated inflammation, further adding support for a negative association between Dolosigranulum 
and S. aureus. Our experiments complement these earlier microbiome association studies and extend these with data on the anti-inflammatory potential of $D$. pigrum against $S$. aureus-induced inflammatory responses.

Furthermore, we could also show that the host interaction and nasal homeostasis-promoting capacity of D. pigrum involves the nasal epithelial barrier function. Barrier-enhancing effects in gut and skin epithelial cells are well documented for several LAB strains (Anderson et al., 2010; O'Neill et al., 2013; Orlando et al., 2014). For the nose, disruptions in epithelial barrier integrity have been described for chronic airway diseases such as CRS and allergic rhinitis, with among others, an important role for $S$. aureus because of its enterotoxins (Martens et al., 2021). Here, it is thus of interest that we found a protective effective of D. pigrum AMBR11 in a murine model of IL-4-induced barrier disruption. To the best of our knowledge, documentation on such barrier-enhancing effects on the URT epithelium is scarce. Recently, we have also found barrier-protective effects for the URT isolate L. casei AMBR2 (Martens et al., 2021). Notably, compared to D. pigrum, L. casei has a weaker association with the nose as its main habitat based on its lower prevalence and relative abundance in the URT (De Boeck et al., 2020) and its association with more habitats as nomadic species (Duar et al., 2017). Both immune signaling and pathogen killing likely play a role in the beneficial action of $D$. pigrum; however, understanding the detailed role of each of these mechanisms would require detailed dedicated research at the molecular level. TLR activation by D. pigrum was also explored in the present study, as these receptors are involved in epithelial barrier functioning, with TLR2/6 activation generally linked to barrier-enhancing and anti-inflammatory signaling effects; however, TLR2/1 is rather involved in inflammation and barrier-disruptive effects (Martens et al., 2021, 2021; Yuki et al., 2011). We observed that D. pigrum strongly activated the TLR2/TLR6 heterodimer, even stronger than L. casei AMBR2 (Martens et al., 2021) and S. aureus, the latter mainly inducing TLR1/6.

Taken together, the data on the anti-inflammatory and barrier-enhancing properties of the health-associated strain D. pigrum AMBR11 are important to substantiate a role for Dolosigranulum as nasal mutualist. The previous documented antimicrobial properties of D. pigrum (Brugger et al., 2020) were insufficient to establish such role, because several pathogens such as Candida albicans and S. aureus can overgrow their habitat by having antimicrobial properties against the resident microbiota, in addition to having toxic or barrier-disruptive effects such as invading target cells. Whether the previously described antimicrobial Dolosigranulum strains derived from a diseased context (Brugger et al., 2020) are also capable of preventing barrier disruption by pathogenic species and effectively protecting against $S$. aureus infection in vivo remains to be established, considering that anti-inflammatory properties are highly variable at the strain level (Lebeer et al., 2018; Lee et al., 2013), but also that isolation from a disease context does not imply a causative role in disease. Nevertheless, from a safety perspective, one could argue that D. pigrum AMBR11 isolated from a healthy person would represent a more appropriate candidate for development into a topical next-generation probiotic or live-biotherapeutic product. Such formulation might be of interest to a broad population, because Dolosigranulum is detected in high abundances, with relative abundance up to $50 \%$ observed in certain individuals, either adults (De Boeck et al., 2019; Brugger et al., 2020; Escapa et al., 2018; Gan et al., 2021; Yan et al., 2013) or children (Biesbroek et al., 2014a, 2014b). A broad range of patients suffering from diverse URT diseases, including CRS in adults or otitis media in children, might benefit from D. pigrum AMBR11 supplementation, but this of course requires extensive safety and efficacy evaluation in follow-up clinical trials.

\section{Limitations of the study}

One limitation of this study is that the suggested D. pigrum AMBR11 mechanisms of action responsible for its observed beneficial effects require more detailed investigation to unravel their exact contribution to nasal homeostasis. The genomic analysis of $D$. pigrum and the different mechanisms proposed in our work pave the way for more dedicated experimental design, and subsequent studies can, for instance, focus on molecular insights that can be obtained via purification of antimicrobial molecules produced by Dolosigranulum. Alternatively, the possibility to construct gene deletion mutants could be investigated, allowing direct comparison of such constructed mutant and its parental strain for relevant phenotypes, thereby directly establishing the relevance of the functionality encoded by the disrupted gene to nasal health. In addition, the exact role of Dolosigranulum on the airway epithelial barrier is an interesting topic for follow-up research to generate more mechanistic insights on, for instance, the tight junction proteins that are involved. Finally, the main focus here was on one isolate, D. pigrum AMBR11. The isolation of other 
healthy isolates and the comparison of their activity with D. pigrum AMBR11 will also help to further understand the role of this microbe in airway health.

\section{STAR $\star$ METHODS}

Detailed methods are provided in the online version of this paper and include the following:

- KEY RESOURCE TABLE

- RESOURCE AVAILABILITY

O Lead contact

O Materials availability

O Data and code availability

- EXPERIMENTAL MODEL AND SUBJECT DETAILS

Human subjects

Microbial strains and culturing methodology

C Cell lines

O Galleria mellonella

O In vivo animal model

- METHOD DETAILS

O Microbiome study and Illumina MiSeq 16S rRNA amplicon sequencing

O Analysis of public data sets for Dolosigranulum

O Phylogenomic and pangenome analyses

$O$ Isolation and whole genome sequencing of Dolosigranulum pigrum AMBR11 and AMBR12 from the healthy URT

Time course analysis of the antimicrobial activity against $S$. aureus and determination of lactic acid concentration in supernatant

O Induction of cytokine gene expression in human Calu-3 cells at mRNA level

HEK-Blue ${ }^{\text {TM }}$ hTLR2/6 and hTLR2/1 induction experiments

O Galleria mellonella survival assay

O In vivo effect of D. pigrum AMBR11 in a murine model of IL-4-induced barrier dysfunction

- QUANTIFICATION AND STATISTICAL ANALYSIS

\section{SUPPLEMENTAL INFORMATION}

Supplemental information can be found online at https://doi.org/10.1016/j.isci.2021.102978.

\section{ACKNOWLEDGMENTS}

This research was funded by a grant from the Flanders Innovation and Entrepreneurship Agency [IWT-SBO ProCure project (IWT/50052)], by the personal grants of I.D.B. 1S17916N and S.W. 11A0618N, and by the European Research Council grant of S.L. (42/FA070500/8330). The authors want to thank the entire research group ENdEMIC of the University of Antwerp. They also want to thank the entire ENT department of the Antwerp University Hospital and University Hospitals of Leuven, the Center of Medical Genetics, and all volunteers that participated. Finally, the authors want to thank all partners of the IWT-SBO ProCure project. Graphical abstract created with BioRender.com.

\section{AUTHOR CONTRIBUTIONS}

Conceived and designed the study: IDB, SL. Designed the experiments: IDB, CA, IS, SL. The experiments were performed by IDB, CA, KM, and EC. Data analysis and interpretation was done by IDB, CA, KM, BS, IS, $E C, J J$ and PAB. Bioinformatics analysis and writing of the scripts by SaW, StW, WvB, and JD. PH and OV were responsible for the clinical assessments and interventions. IDB and SL wrote the first draft, PAB revised the manuscript and all authors read the final version.

\section{DECLARATION OF INTERESTS}

A patent application (PCT/EP2019/086763) has been filed on 20 December 2019 related to this work. P.B. is a consultant for multiple companies in the food and health industry, but they were not involved in this manuscript. The remaining authors have no conflicts of interest to declare. 
INCLUSION AND DIVERSITY

We worked to ensure gender balance in the recruitment of human subjects. We worked to ensure that the study questionnaires were prepared in an inclusive way.

Received: May 7, 2021

Revised: July 28, 2021

Accepted: August 10, 2021

Published: September 24, 2021

\section{REFERENCES}

Afzal, M.I., Jacquet, T., Delaunay, S., Borges, F. Millière, J.B Revol-Junelles, A.M, and CailliezGrimal, C. (2010). Carnobacterium maltaromaticum: identification, isolation tools, ecology and technological aspects in dairy products. Food Microbiol. 27, 573-579.

Aguirre, M., Morrison, D., Cookson, B.D., Gay, F.W., and Collins, M.D. (1993). Phenotypic and phylogenetic characterization of some Gemella-like organisms from human infections: description of Dolosigranulum pigrum gen. nov., sp. nov. J. Appl. Bacteriol. $75,608-612$

Alakomi, H.L., Skyttä, E., Saarela, M., MattilaSandholm, T., Latva-Kala, K., and Helander, I.M. (2000). Lactic acid permeabilizes gramnegative bacteria by disrupting the outer membrane. Appl. Environ. Microbiol. 66 2001-2005.

Anderson, R.C., Cookson, A.L., McNabb, W.C. Park, Z., McCann, M.J. Kelly, W.J., and Roy, N.C. (2010). Lactobacillus plantarum MB452 enhances the function of the intestinal barrie by increasing the expression levels of genes involved in tight junction formation. BMC Microbiol. 10, 316

Biesbroek, G., Tsivtsivadze, E., Sanders, E.a M. Montijn, R., Veenhoven, R.H., Keijser, B.J.F., and Bogaert, D. (2014a). Early respiratory microbiota composition determines bacterial succession patterns and respiratory health in children. Am. J. Respir. Crit. Care Med. 190, 1283-1292.

Biesbroek, G., Bosch, A.a T.M. Wang, X. Keijser B.J.F., Veenhoven, R.H., Sanders, E.a M., and Bogaert, D. (2014b). The impact of breastfeeding on nasopharyngeal microbial communities in infants. Am. J. Respir. Crit. Care Med. 190, 298-308.

De Boeck, I., Wittouck, S., Wuyts, S., Oerlemans, E.F.M., van den Broek, M.F.L., Vandenheuvel, D., Vanderveken, O., and Lebeer, S. (2017).

Comparing the healthy nose and nasopharynx microbiota reveals continuity as well As nichespecificity. Front. Microbiol. 8, 2372.

De Boeck, I., Wittouck, S., Martens, K., Claes, J., Jorissen, M. Steelant, B., van den Broek, M.F.L., Seys, S.F., Hellings, P.W., Vanderveken, O.M. et al. (2019). Anterior nares diversity and pathobionts represent sinus microbiome in chronic rhinosinusitis. MSphere 4, e00532-19.

De Boeck, I., van den Broek, M.F.L., Allonsius, C.N., Spacova, I. Wittouck, S., Martens, K. Wuyts, S., Cauwenberghs, E., Jokicevic, K.,
Vandenheuvel, D., et al. (2020). Lactobacilli have a niche in the human nose. Cell Rep. 31, 107674.

Bosch, A.A.T.M., Levin, E., van Houten, M.A Hasrat, R., Kalkman, G., Biesbroek, G., de Steenhuijsen Piters, W.A.A., de Groot, P.-K.C.M Pernet, P., Keijser, B.J.F., et al. (2016) Development of upper respiratory tract microbiota in infancy is affected by mode of delivery. EBioMedicine 9, 336-345.

van den Broek, M.F.L., de Boeck, I., Claes, I.J.J. Nizet, V., and Lebeer, S. (2018). Multifactoria inhibition of lactobacilli against the respiratory tract pathogen Moraxella catarrhalis. Benef. Microbes 9, 429-439.

Brugger, S.D., Eslami, S.M., Pettigrew, M.M., Escapa, I.F., Henke, M.M., Kong, Y., and Lemon, K.P. (2020). Dolosigranulum pigrum cooperation and competition in human nasal microbiota. MSphere 5, e00852.

Bustin, S.A., Benes, V., Garson, J.A., Hellemans, J. Huggett, J., Kubista, M. Mueller, R. Nolan, T. Pfaffl, M.W., Shipley, G.L., et al. (2009). The MIQE guidelines: minimum information for publication of quantitative real-time PCR experiments. Clin. Chem. 55, 611-622.

Capella-Gutiérrez, S., Silla-Martínez, J.M., and Gabaldón, T. (2009). trimAl: a tool for automated alignment trimming in large-scale phylogenetic analyses. Bioinformatics 25, 1972-1973.

Chandrabalan, A., Thibeault, P.E., DeinhardtEmmer, S., Nordengrün, M., labal, J., Mrochen, D.M., Mittmann, L.A., Chamling, B., Reichel, C.A. Ramachandran, $R$, et al (2021). S aureus-serine protease-like protein B (SplB) activates PAR2 and induces endothelial barrier dysfunction. BioRxiv. https://doi.org/10.1101/2020.12.30.424670.

Consortium, T.H.M.P., Huttenhower, C., Gevers, D. Knight, R Abubucker, S, Badger, J.H. Chinwalla, A.T., Creasy, H.H., Earl, A.M., FitzGerald, M.G., et al. (2012). Structure, function and diversity of the healthy human microbiome. Nature 486, 207-214.

Derycke, L., Pérez-Novo, C., Van Crombruggen, K., Corriveau, M.N., and Bachert, C. (2010). Staphylococcus aureus and chronic airway disease. World Allergy Organ. J. 3, 223-228.

Du Toit, M., Huch, M., Cho, G.S., and Franz, C.M.A.P. (2014). The genus Streptococcus. In Lactic Acid Bacteria: Biodiversity and Taxonomy, W.H. Holzapfel and B.J.B. Wood, eds. (WileyBlackwell).

Duar, R.M. Lin X. B Z Zheng, J Martino, M.E. Grenier, T., Pérez-Muñoz, M.E., Leulier, F.,
Gänzle, M., and Walter, J. (2017). Lifestyles in transition: evolution and natural history of the genus Lactobacillus. FEMS Microbiol. Rev. 41, S27-S48.

Eloe-Fadrosh, E.A., and Rasko, D.A. (2013). The human microbiome: from symbiosis to pathogenesis. Annu. Rev. Med. 64, 145-163.

Escapa, I.F., Chen, T., Huang, Y., Gajare, P. Dewhirst, F.E., and Lemon, K.P. (2018). New insights into human nostril microbiome from the expanded human oral microbiome database (eHOMD): a resource for the microbiome of the human aerodigestive tract. MSystems 3, e00187.

Faden, H., and Dryja, D. (1989). Recovery of a unique bacterial organism in human middle ear fluid and its possible role in chronic otitis media. J. Clin. Microbiol. 27, 2488-2491.

Gan, W., Yang, F., Meng, J., Liu, F., Liu, S., and Xian, J. (2021). Comparing the nasal bacterial microbiome diversity of allergic rhinitis, chronic rhinosinusitis and control subjects. Eur. Arch. Otorhinolaryngol. 278, 711-718.

Gan, W., Yang, F., Tang, Y., Zhou, D., Qing, D. Hu, J., Liu, S., Liu, F., and Meng, J. (2019). The difference in nasal bacterial microbiome diversity between chronic rhinosinusitis patients with polyps and a control population. Int. Forum Allergy Rhinol. 9, 582-592.

Gilbert, J.A., Jansson, J.K., and Knight, R. (2014) The Earth Microbiome project: successes and aspirations. BMC Biol. 12, 69.

Hasegawa, K., Mansbach, J.M., Ajami, N.J. Espinola, J.A., Henke, D.M., Petrosino, J.F., Piedra, P.A., Shaw, C.A., Sullivan, A.F., and Camargo, C.A. (2016). Association of nasopharyngeal microbiota profiles with bronchiolitis severity in infants hospitalised for bronchiolitis. Eur. Respir. J. 48, 1329-1339.

Hellings, P.W., and Steelant, B. (2020). Epithelia barriers in allergy and asthma. J. Allergy Clin. Immunol. 145, 1499-1509.

Hoang, D.T., Chernomor, O., Von Haeseler, A., Minh, B.Q., and Vinh, L.S. (2018). UFBoot2: improving the ultrafast bootstrap approximation. Mol. Biol. Evol. 35, 518-522.

Hyatt, D., Chen, G.L., LoCascio, P.F., Land, M.L., Larimer, F.W., and Hauser, L.J. (2010). Prodigal: prokaryotic gene recognition and translation initiation site identification. BMC Bioinformatics $11,1-11$.

Kalyaanamoorthy, S., Minh, B.Q., Wong, T.K.F. Von Haeseler, A., and Jermiin, L.S. (2017). 
ModelFinder: fast model selection for accurate phylogenetic estimates. Nat. Methods 14 587-589.

De Keersmaecker, S.C.J., Verhoeven, T.L.A., Desair, J., Marchal, K., Vanderleyden, J., and Nagy, I. (2006). Strong antimicrobial activity of Lactobacillus rhamnosus GG against Salmonella typhimurium is due to accumulation of lactic acid. FEMS Microbiol. Lett. 259, 89-96.

Kirchberger, P.C., Schmidt, M.L., and Ochman, H. (2020). The ingenuity of bacterial genomes. Annu. Rev. Microbiol. 74, 815-834.

Laclaire, L., and Facklam, R. (2000). Antimicrobial susceptibility and clinical sources of

Dolosigranulum pigrum cultures. Antimicrob.

Agents Chemother. 44, 2001-2003.

Lane, D.J. (1991). 16S/23S rRNA sequencing. In Nucleic Acid Techniques in Bacterial Systematics, E. Stackebrandt and M. Goodfellow, eds. (John Wiley \& Sons), pp. 115-175.

Lappan, R., Imbrogno, K., Sikazwe, C., Anderson, D., Mok, D., Coates, H., Vijayasekaran, S. Bumbak, P., Blyth, C.C., Jamieson, S.E., et al. (2018). A microbiome case-control study of recurrent acute otitis media identified potentially protective bacterial genera. BMC Microbiol. 18, 13.

Laufer, A.S., Metlay, J.P., Gent, J.F., Fennie, K.P., Kong, Y., and Pettigrew, M.M. (2011). Microbial communities of the upper respiratory tract and otitis media in children. MBio 2, e00245-10.

Lawson, P.A., and Caldwell, M.E. (2014). The family Carnobacteriaceae. In The Prokaryotes, E. Rosenberg, E.F. DeLong, E. Stackebrandt, and F. Thompson, eds. (Springer Berlin Heidelberg) pp. 19-65.

Lebeer, S., Bron, P.A., Marco, M.L., Van Pijkeren, J.-P. O', M., Motherway, C. Hill, C. Pot, B., Roos, S., Klaenhammer, T., et al. (2018). Identification of probiotic effector molecules: present state and future perspectives. Curr. Opin. Biotechnol. 49, 217-223.

Lee, I.C., Tomita, S., Kleerebezem, M., and Bron, P.A. (2013). The quest for probiotic effector molecules - unraveling strain specificity at the molecular level. Pharmacol. Res. 69, 61-74.

Liu, B., Zheng, D., Jin, O., Chen, L., and Yang, J. (2019). Vfdb 2019: a comparative pathogenomic platform with an interactive web interface. Nucleic Acids Res. 47, D687-D692.

Macklaim, J.M., Gloor, G.B., Anukam, K.C., Cribby, S., and Reid, G. (2011). At the crossroads of vaginal health and disease, the genome sequence of Lactobacillus iners AB-1. Proc. Natl. Acad. Sci. U S A 108, 4688-4695.

Man, W.H., de Steenhuijsen Piters, W.A.A., and Bogaert, D. (2017). The microbiota of the respiratory tract: gatekeeper to respiratory health. Nat. Rev. Microbiol. 15, 259-270.

Martens, K., De Boeck, I., Jokicevic, K., Kiekens, F., Farré, R., Vanderveken, O.M. Seys, S.F., and Lebeer, S. (2021). Lacticaseibacillus casei AMBR2 restores airway epithelial integrity in chronic rhinosinusitis with nasal polyps. Allergy Asthma, Immunol. Res. 13, 560-575.
Martens, K., Seys, S.F., Alpizar, Y.A., Schrijvers, R., Bullens, D.M.A., Breynaert, C., Lebeer, S., and Steelant, B. (2021). Staphylococcus aureus enterotoxin B disrupts nasal epithelial barrier integrity. Clin. Exp. Allergy 51, 87-98.

Martina, F., Perrotta, F., Nicolai, A., Maffucci, R., Pratillo, A. Mollica, M. Bianco, A., and Calabrese, C. (2019). Staphylococcus Aureus in chronic airway diseases: an overview. Respir. Med. 155, 66-71.

McDonald, D., Clemente, J.C., Kuczynski, J., Rideout, J.R., Stombaugh, J., Wendel, D., Wilke, A., Huse, S., Hufnagle, J., Meyer, F., et al. (2012) The Biological Observation Matrix (BIOM) forma or: how I learned to stop worrying and love the ome-ome. Gigascience 1, 7.

Moretti, S., Smets, W., Hofman, J., Mubiana, K.V. Oerlemans, E., Vandenheuvel, D., Samson, R. Blust, R., and Lebeer, S. (2019). Human inflammatory response of endotoxin affected by particulate matter-bound transition metals. Environ. Pollut. 244, 118-126.

Nayfach, S., Roux, S. Seshadri, R., Udwary, D. Varghese, N., Schulz, F., Wu, D., Paez-espino, D. Chen, I., Huntemann, M., et al. (2020). A genomic catalog of Earth 's microbiomes. Nat. Biotechnol. 39, 499-509.

Nguyen, L.T., Schmidt, H.A., Von Haeseler, A. and Minh, B.Q. (2015). IQ-TREE: a fast and effective stochastic algorithm for estimating maximum-likelihood phylogenies. Mol. Biol. Evol. $32,268-274$.

O'Neill, C.a., Sultana, R., and McBain, A.J. (2013) Strain-dependent augmentation of tight-junction barrier function in human primary epidermal keratinocytes by lactobacillus and

bifidobacterium lysates. Appl. Environ. Microbiol. $79,4887-4894$

Oh. P.L., Benson, A.K., Peterson, D.A., Patil, P. B. Moriyama, E.N., Roos, S., and Walter, J. (2010). Diversification of the gut symbiont lactobacillus reuteri as a result of host-driven evolution. ISME J 4, 377-387.

Orlando, A., Linsalata, M., Notarnicola, M. Tutino, V., and Russo, F. (2014). Lactobacillus GG restoration of the gliadin induced epithelial barrier disruption: the role of cellular polyamines BMC Microbiol. 14, 19

Parks, D.H., Chuvochina, M., Chaumeil, P.A. Rinke, C., Mussig, A.J., and Hugenholtz, P. (2020) A complete domain-to-species taxonomy for Bacteria and Archaea. Nat. Biotechnol. 38, 1079 1086.

Pasolli, E., Schiffer, L., Manghi, P., Renson, A., Obenchain, V., Truong, D.T., Beghini, F., Malik, F. Ramos, M., Dowd, J.B., et al. (2017). Accessible, curated metagenomic data through ExperimentHub. Nat. Methods 14, 1023-1024.

Perez, R.H., Zendo, T., and Sonomoto, K. (2014). Novel bacteriocins from lactic acid bacteria (LAB): various structures and applications. Microb. Cell Fact. 13, S3.

Petrova, M.I., Lievens, E., Malik, S., Imholz, N., and Lebeer, S. (2015). Lactobacillus species as biomarkers and agents that can promote various aspects of vaginal health. Front. Physiol. 6, 81.
R Core Team (2020). R: A Language and Environment for Statistical Computing.

De Rudder, C., Garcia-Tímermans, C., De Boeck, I., Lebeer, S., Van de Wiele, T., and Calatayud Arroyo, M. (2020). Lacticaseibacillus casei AMBR2 modulates the epithelial barrier function and immune response in a donor-derived nasal microbiota manner. Sci. Rep. 10, 1-16.

Schliep, K., Potts, J.A., Morrison, A.D., and Grimm, W.G. (2017). Intertwining phylogenetic trees and networks. Methods Ecol. Evol. 8, 12121220.

Steelant, B., Farré, R., Wawrzyniak, P., Belmans J., Dekimpe, E., Vanheel, H., Van Gerven, L., Kortekaas Krohn, I., Bullens, D.M.A., Ceuppens, J.L., et al. (2016). Impaired barrier function in patients with house dust mite-induced allergic rhinitis is accompanied by decreased occludin and zonula occludens-1 expression. J. Allergy Clin. Immunol. 137, 1043-1053.e5.

Steelant, B., Seys, S.F., Van Gerven, L., Van Woensel, M., Farré, R., Wawrzyniak, P., Kortekaas Krohn, I., Bullens, D.M., Talavera, K., Raap, U., et al. (2018). Histamine and T helper cytokinedriven epithelial barrier dysfunction in allergic rhinitis. J. Allergy Clin. Immunol. 141, 951-963.e8.

Szkaradkiewicz, A., Karpiński, T.M., Zeidler, A Szkaradkiewicz, A.K., Masiuk, H., and GiedrysKalemba, S. (2012). Cytokine response in patients with chronic infections caused by Staphylococcus aureus strains and diversification of their Agr system classes. Eur. J. Clin. Microbiol. Infect. Dis. 31, 2809-2815.

Teufelberger, A.R., Bröker, B.M., Krysko, D.V., Bachert, C., and Krysko, O. (2019)

Staphylococcus aureus orchestrates type 2 airway diseases. Trends Mol. Med. 25, 696-707.

Thompson, L.R., Sanders, J.G., McDonald, D., Amir, A., Ladau, J., Locey, K.J., Prill, R.J., Tripathi, A., Gibbons, S.M., Ackermann, G., et al. (2017). A communal catalogue reveals Earth's multiscale microbial diversity. Nature 551, 457-463.

Turner, S., Pryer, K.M., Miao, V.P.W., and Palmer, J.D. (1999). Investigating deep phylogenetic relationships among cyanobacteria and plastids by small subunit rRNA sequence analysis. J. Eukaryot. Microbiol. 46, 327-338.

Vandesompele, J., De Preter, K., Pattyn, F., Poppe, B., Van Roy, N., De Paepe, A., and Speleman, F. (2002). Accurate normalization of real-time quantitative RT-PCR data by geometric averaging of multiple internal control genes. Genome Biol. 3, research0034.1research0034.11.

Wickham, H., Averick, M., Bryan, J., Chang, W., McGowan, L.D., François, R., Grolemund, G., Hayes, A., Henry, L., Hester, J., et al. (2019). Welcome to the \{tidyverse\}. J. Open Source Softw. 4, 1686.

Wittouck, S. (2020a). Tidyamplicons: Functions to Manipulate and Visualize Amplicon Abundance Data (GitHub).

Wittouck, S. (2020b). Tidygenomes: A Grammar of Genome Data Manipulation (GitHub).

Wittouck, S., Wuyts, S., Meehan, C.J., van Noort, V., and Lebeer, S. (2019). A genome-based 
species taxonomy of the lactobacillus genus complex. MSystems 4, e00264-19.

Wuyts, S., Wittouck, S., De Boeck, I., Allonsius, C.N., Pasolli, E., Segata, N., and Lebeer, S. (2017) Large-Scale phylogenomics of the lactobacillus casei group highlights taxonomic inconsistencies and reveals novel clade-associated features. MSystems 2, e00061-17.

Xiao, C., Puddicombe, S.M., Field, S., Haywood, J., Broughton-Head, V., Puxeddu, I., Haitchi,

H.M. Vernon-Wilson, E. Sammut, D. Bedke, N., et al. (2011). Defective epithelial barrier function in asthma. J. Allergy Clin. Immunol. $128,549$.
Yan, M., Pamp, S.J., Fukuyama, J., Hwang, P.H., Cho, D.-Y., Holmes, S., and Relman, D.A. (2013). Nasal microenvironments and interspecific interactions influence nasal microbiota complexity and S. aureus carriage. Cell Host Microbe 14, 631-640.

Yu, G. Smith, D.K., Zhu, H., Guan, Y., and Lam, T.T.Y. (2017). ggtree: an r package for visualization and annotation of phylogenetic trees with their covariates and other associated data. Methods Ecol. Evol. 8, 28-36.

Yuki, T., Yoshida, H., Akazawa, Y., Komiya, A., Sugiyama, Y., and Inoue, S. (2011). Activation of TLR2 enhances tight junction barrier in epiderma keratinocytes. J. Immunol. 187, 3230-3237.
Zhao, R., Guo, Z., Zhang, R., Deng, C., Xu, J., Dong, W., Hong, Z, Yu, H., Situ, H., Liu, C., et al. (2018). Nasal epithelial barrier disruption by particulate matter $\leq 2.5 \mu \mathrm{m}$ via tight junction protein degradation. J. Appl. Toxicol. 38 678-687.

Zheng, J., Wittouck, S., Salvetti, E., Franz C.M.A.P. Harris, H.M.B. Mattarelli, P. O'Toole P.W., Pot, B., Vandamme, P., Walter, J., et al. (2020). A taxonomic note on the genus Lactobacillus: description of 23 novel genera, emended description of the genus Lactobacillus Beijerinck 1901, and union of Lactobacillaceae and Leuconostocaceae. Int. J. Syst. Evol. Microbiol. 70, 2782-2858. 


\section{STAR $\star$ METHODS}

\section{KEY RESOURCE TABLE}

\begin{tabular}{|c|c|c|}
\hline REAGENT or RESOURCE & SOURCE & IDENTIFIER \\
\hline \multicolumn{3}{|l|}{ Bacterial strains } \\
\hline Lactobacillus rhamnosus GG & ATCC & ATCC53103 \\
\hline Lactobacillus casei AMBR2 & $\begin{array}{l}\text { LAMB } \\
\text { BCCM/LMG P-30039 }\end{array}$ & ENA: Study PRJEB21025, accession GCA_900185125 \\
\hline Dolosigranulum pigrum AMBR11 & LAMB BCCM/LMG P-31124 & ENA: Study PRJEB32716, accession GCA_901830375 \\
\hline Dolosigranulum pigrum AMBR12 & LAMB BCCM/LMG P-31154 & ENA: Study PRJEB32716, accession GCA_905071805 \\
\hline Dolosigranulum pigrum LMG15126 & & NZ_AGEF00000000.1 \\
\hline Staphylococcus aureus & ATCC & ATCC29213 \\
\hline \multicolumn{3}{|l|}{ Biological Samples } \\
\hline adult human nasopharyngeal swabs & $\begin{array}{l}\text { University of Antwerp, } \\
\text { University Hospitals of Leuven }\end{array}$ & N/A \\
\hline \multicolumn{3}{|l|}{ Chemicals, Peptides, and Recombinant Proteins } \\
\hline de Man, Rogosa and Sharpe (MRS) & Difco & BD288210 \\
\hline Mueller Hinton & LabM limited & LAB039-A \\
\hline Brain Hart Infusion (BHI) & International Medical Products & 8532134 \\
\hline Tween 80 (polysorbate) & Carl Roth Belgium & 9139.1 \\
\hline Sheep blood defibrinated & bioTRADING & BTSG100 \\
\hline Tryptic Soy Agar & Merck Life Science B.V. (Sigma) & 22091-500G \\
\hline Minimal Essential Medium (MEM) & Life Technologies & $31095-029$ \\
\hline Fetal Calf Serum (FCS) & Hyclone & 12350273 \\
\hline Penicillin-streptomycin (100U/mL) & Gibco & 15140122 \\
\hline Trypsin EDTA (0.25\%) & Gibco & 25200072 \\
\hline Dulbecco's Modified Eagle Medium (DMEM) & Life technologies & 31053028 \\
\hline Lysozyme & Sigma Aldrich & L6876-5G \\
\hline mutanolysin $(100 \mathrm{U} / \mathrm{mL})$ & Sigma Aldrich & M9901-10KU \\
\hline PBS & Gibco & 14040-091 \\
\hline Readyscript ${ }^{\circledR}$ cDNA synthesis mix & Sigma Aldrich & RDRT-500RXN \\
\hline PowerSYBR $\circledast$ Green PCR Master Mix & Applied Biosystems & 13266519 \\
\hline $0.1 \%$ hexetidine $\left(\right.$ Hextril $\left.{ }^{\circledR}\right)$ & Famar Orléans & N/A \\
\hline \multicolumn{3}{|l|}{ Critical Commercial Assays } \\
\hline NucleoSpin 96 Tissue kit & Machery-Nagel & MN 740609.50 \\
\hline Nextera XT DNA Sample Preparation kit & Illumina & TG-131-1096 \\
\hline QIAamp Powerfecal DNA kit & Qiagen & $12830-50$ \\
\hline Agencourt AMPure XP & Beckman Coulter & A63881 \\
\hline RNeasy Mini kit & Qiagen & 74104 \\
\hline Roche Yellow line kit D/L lactic acid & Roche & 11112821035 \\
\hline \multicolumn{3}{|l|}{ Deposited Data } \\
\hline Lactobacillus casei AMBR2 & (Wuyts et al., 2017) & ENA: Study PRJEB21025, accession GCA_900185125 \\
\hline Dolosigranulum pigrum AMBR11 & This paper & ENA: Study PRJEB32716, accession GCA_901830375 \\
\hline Dolosigranulum pigrum AMBR12 & This paper & ENA:Study PRJEB32716, accession GCA_905071805 \\
\hline DNA-Seq data study B300201524257 & (De Boeck et al., 2017, 2019) & ENA: PRJEB23057, PRJEB30316 \\
\hline
\end{tabular}




\begin{tabular}{|c|c|c|}
\hline \multicolumn{3}{|l|}{ Continued } \\
\hline REAGENT or RESOURCE & SOURCE & IDENTIFIER \\
\hline \multicolumn{3}{|l|}{ Experimental Models: Cell Lines } \\
\hline Calu-3 & ATCC & HTB-55 \\
\hline HEK-Blue ${ }^{\text {TM }}$ hTLR2-TLR1 & Invivogen & Cat code: hkb-ht|r21 \\
\hline HEK-Blue ${ }^{T M}$ hTLR2-TLR6 & Invivogen & Cat code: hkb-htlr26 \\
\hline \multicolumn{3}{|l|}{ Experimental models: Organisms/strains } \\
\hline Galleria mellonella & Anaconda reptiles (Kontich, Belgium) & $\mathrm{N} / \mathrm{A}$ \\
\hline $\mathrm{BALB} / \mathrm{c}$ mice & Envigo (Horst, The Netherlands) & $\mathrm{N} / \mathrm{A}$ \\
\hline \multicolumn{3}{|l|}{ Oligonucleotides } \\
\hline $\begin{array}{l}\text { 16S rRNA gene (27F) } \\
\text { AGAGTTTGATCMTGGCTCAG }\end{array}$ & (Lane, 1991) & N/A \\
\hline \multicolumn{3}{|l|}{ GGTTACCTTGTTACGACTT } \\
\hline $\begin{array}{l}\text { CYC1-F (qPCR) } \\
\text { CATGTCCCAGATAGCCAAGGA }\end{array}$ & (Moretti et al., 2019) & N/A \\
\hline $\begin{array}{l}\text { CYC1-R (qPCR) } \\
\text { CTTGTGCCGCTTTATGGTGTAG }\end{array}$ & (Moretti et al., 2019) & N/A \\
\hline $\begin{array}{l}\text { ATP5B-F (qPCR) } \\
\text { GCAGGAAAGAATTACCACTACCAAG }\end{array}$ & (Moretti et al., 2019) & $\mathrm{N} / \mathrm{A}$ \\
\hline $\begin{array}{l}\text { ATP5B-R (qPCR) } \\
\text { TGGTAGCATCCAAATGGGCAA }\end{array}$ & (Moretti et al., 2019) & N/A \\
\hline $\begin{array}{l}\text { IL1 } \beta \text {-F (qPCR) } \\
\text { TTGCTCAAGTGTCTGAAGCAGC }\end{array}$ & (Moretti et al., 2019) & N/A \\
\hline $\begin{array}{l}\text { IL1 } \beta-R \text { (qPCR) } \\
\text { CAAGTCATCCTCATTGCCACTG }\end{array}$ & (Moretti et al., 2019) & $\mathrm{N} / \mathrm{A}$ \\
\hline $\begin{array}{l}\text { IL8-F (qPCR) } \\
\text { TGGCAGCCTTCCTGATTTCT }\end{array}$ & (Moretti et al., 2019) & N/A \\
\hline $\begin{array}{l}\text { IL8-R (qPCR) } \\
\text { TTAGCACTCCTTGGCAAAACTG }\end{array}$ & (Moretti et al., 2019) & N/A \\
\hline \multicolumn{3}{|l|}{ ССTCTGATGGCACCACCAG } \\
\hline \multicolumn{3}{|l|}{ TCTTCTCGAACCCCGAGTGA } \\
\hline \multicolumn{3}{|l|}{ Software and Algorithms } \\
\hline GraphPad Prism & GraphPad Software & https://www.graphpad.com/ \\
\hline DADA2, version 1.6 .0 & https://doi.org/10.1038/nmeth.3869 & https://benjjneb.github.io/dada2/index.html \\
\hline R version 3.6.3 (R Core Team, 2020) & R Core Team (2020) & https://www.r-project.org/ \\
\hline Tidyamplicons & (Wittouck, 2020a) & github.com/Swittouck/tidyamplicons \\
\hline Phyloseq & $\begin{array}{l}\text { https://doi.org/10.1371/ } \\
\text { journal.pone.0061217 }\end{array}$ & https://github.com/joey711/phyloseq \\
\hline Biom python package & (McDonald et al., 2012) & $\begin{array}{l}\text { https://biom-format.org/documentation/ } \\
\text { biom_format.html }\end{array}$ \\
\hline Genome Taxonomy Database (GTDB) & (Parks et al., 2020) & https://gtdb.ecogenomic.org/ \\
\hline Prodigal v2.6.3 & (Hyatt et al., 2010) & https://github.com/hyattpd/Prodigal/releases \\
\hline Progenomics version $13 \mathrm{~b} 9 \mathrm{be} 1$ & (Wittouck et al., 2019) & https://github.com/SWittouck/SCARAP \\
\hline trimal v1.4.rev15 & (Capella-Gutiérrez et al., 2009) & https://github.com/scapella/trimal \\
\hline IQTREE v1.6.11 & $\begin{array}{l}\text { (Hoang et al., 2018; Kalyaanamoorthy } \\
\text { et al., 2017; Nguyen et al., 2015) }\end{array}$ & http://www.iqtree.org/ \\
\hline
\end{tabular}




\begin{tabular}{|c|c|c|}
\hline \multicolumn{3}{|l|}{ Continued } \\
\hline REAGENT or RESOURCE & SOURCE & IDENTIFIER \\
\hline ggtree v2.0.2 & (Yu et al., 2017) & $\begin{array}{l}\text { https://bioconductor.org/packages/release/ } \\
\text { bioc/html/ggtree.html }\end{array}$ \\
\hline tidyverse v1.3.0 & (Wickham et al., 2019) & $\begin{array}{l}\text { https://www.tidyverse.org/blog/2019/11/ } \\
\text { tidyverse-1-3-0/ }\end{array}$ \\
\hline tidygenomes v0.1.3 & (Wittouck, 2020b) & $\begin{array}{l}\text { https://github.com/SWittouck/tidygenomes/ } \\
\text { releases }\end{array}$ \\
\hline phangorn v2.5.5 & (Schliep et al., 2017) & $\begin{array}{l}\text { https://cran.r-project.org/web/packages/ } \\
\text { phangorn/index.html }\end{array}$ \\
\hline qbase ${ }^{+}$ & (Vandesompele et al., 2002) & https://www.qbaseplus.com/ \\
\hline Virulence Factor Database (VFDB) & (Liu et al., 2019) & http://www.mgc.ac.cn/VFs/ \\
\hline Script for EMP data exploration & This paper & $\begin{array}{l}\text { https://github.com/swittouck/ } \\
\text { dolosigranulum_pigrum }\end{array}$ \\
\hline Script for phylogenomic and pangenome analysis & This paper & $\begin{array}{l}\text { https://github.com/swittouck/ } \\
\text { dolosigranulum_pigrum }\end{array}$ \\
\hline \multicolumn{3}{|l|}{ Other } \\
\hline MiSeq Desktop sequencer & Illumina & (M00984, Illumina) \\
\hline Synergy HTX multi-mode reader & Biotek & N/A \\
\hline Take3 & Biotek & N/A \\
\hline StepOne Plus Real-Time PCR System (v.2.0) & Applied Biosystems & N/A \\
\hline
\end{tabular}

\section{RESOURCE AVAILABILITY}

\section{Lead contact}

Further information and requests for resources and reagents should be directed to and will be fulfilled by the Lead Contact, Sarah Lebeer (sarah.lebeer@uantwerpen.be).

\section{Materials availability}

All unique/stable reagents generated in this study are available from the Lead Contact with a completed Materials Transfer Agreement.

\section{Data and code availability}

- All sequencing data were deposited in ENA (https://www.ebi.ac.uk/ena) under accession numbers PRJEB23057 and PRJEB30316.

- D. pigrum AMBR11 and D. pigrum AMBR12 were deposited in ENA under accession number GCA_901830375 and GCA_905071805.

- All scripts for the EMP data exploration can be found at https://github.com/swittouck/ dolosigranulum_pigrum.

- All scripts for the phylogenomic and pangenome analyses can be found at https://github.com/ swittouck/dolosigranulum_pigrum.

\section{EXPERIMENTAL MODEL AND SUBJECT DETAILS}

\section{Human subjects}

Nasopharyngeal swabs from human subjects, aged between 18 and 65 years, were collected in a standardized way by the responsible ear, nose, and throat (ENT) specialist from healthy participants $(n=$ 100, 39\% male, average age 34 years) and CRS patients ( $n=225,63 \%$ male, average age 42 years) at the University of Antwerp, the Antwerp University Hospital and the University Hospital of Leuven between 2015 and 2018, as previously described (De Boeck et al., 2017, 2019). The study was approved by the Ethics Committee of the Antwerp University Hospital/University of Antwerp (B300201524257) 
(ClinicalTrials.gov Identifier: NCT02933983). Written informed consent was obtained from all participants prior to sampling.

\section{Microbial strains and culturing methodology}

Microbial strains used are listed in the key resources table. Dolosigranulum strains were grown at $37^{\circ} \mathrm{C}$ under shaking conditions in $\mathrm{BHI}$ broth, supplemented with $0.5 \%$ Tween 80 . Lactobacilli were grown at $37^{\circ} \mathrm{C}$ without agitation in de Man, Rogosa and Sharpe (MRS) broth (Difco). S. aureus was grown at $37^{\circ} \mathrm{C}$ without agitation in Mueller Hinton (MH) broth (LabM Limited).

\section{Cell lines}

The Calu-3 ATCC $\circledast \mathrm{HTB}-55^{\mathrm{TM}}$ (purchased from ATCC) cell line was cultured at $37^{\circ} \mathrm{C}$ with $5 \% \mathrm{CO}_{2}$ and $90 \%$ relative humidity in $75 \mathrm{~cm}^{2}$ cell tissue flasks containing $20 \mathrm{~mL}$ Minimal Essential Medium (MEM) (Life technologies) supplemented with $10 \%$ heat inactivated fetal calf serum (FCS) (Hyclone) and penicillin-streptomycin $(100 \mathrm{U} / \mathrm{mL})$ (Life technologies). Every three to four days, the culture medium was changed and cells were reseeded when $70-80 \%$ confluency was reached at a 1:2 split ratio using a $0.25 \%$ trypsin-EDTA solution (Life Technologies). Calu-3 cells were seeded at a density of $3 \times 10^{5}$ cells $/ \mathrm{cm}^{2}\left(1.1 \times 10^{6} \mathrm{cell} / \mathrm{sL}\right)$ in 12- or 24-well culture plates (Cellstar) for adhesion and immunomodulation experiments, respectively. Approximately one week after seeding, confluent monolayers were obtained. One day before each experiment, medium was replaced to MEM without any supplements.

HEK-Blue ${ }^{\text {TM }}$ hTLR2-TLR1 and the HEK-Blue ${ }^{\text {TM }} \mathrm{hTLR} 2-$ TLR6 reporter cell line (Invivogen) were cultured at $37^{\circ} \mathrm{C}$ with $5 \% \mathrm{CO}_{2}$ and $90 \%$ relative humidity in $75 \mathrm{~cm}^{2}$ cell tissue flasks containing $20 \mathrm{~mL}$ in Dulbecco's Modified Eagle Medium (DMEM). Prior to an experiment, cells were seeded in a 96-well plate at a concentration of $2.5 \times 10^{5}$ cells/well and incubated for 48 hours at $37^{\circ} \mathrm{C}$ with $5 \% \mathrm{CO}_{2}$.

\section{Galleria mellonella}

G. mellonella were purchased from Anaconda reptiles (Kontich, Belgium) in their final larval stage. Upon arrival, the larvae were stored at $4^{\circ} \mathrm{C}$ and used within 7 days. Fifteen randomly selected larvae were used per group.

\section{In vivo animal model}

Male BALB/c mice (6-8 weeks) were obtained from Envigo (Horst, The Netherlands) and were kept under conventional conditions at the Animal facility of KU Leuven. Experimental procedures were approved by the Ethical Committee for Animal Research at the KU Leuven (P150/2017).

\section{METHOD DETAILS}

\section{Microbiome study and Illumina MiSeq 16S rRNA amplicon sequencing}

Samples were processed, sequenced and analyzed as previously described (De Boeck et al., 2017). Briefly, dual-index paired-end sequencing was performed on the V4 region of the 16S rRNA gene on a MiSeq Desktop sequencer (M00984, Illumina) at the Centre of Medical Genetics, University of Antwerp, Belgium. After sequencing, raw sequencing reads were filtered and denoised using DADA2 (v 1.1.6). Reads with more than 2 expected errors were removed. All sequencing data were deposited in ENA under accession numbers PRJEB23057 and PRJEB30316.

\section{Analysis of public data sets for Dolosigranulum}

Processed OTU-table and sample metadata from the Human Microbiome Project (HMPv35) (Consortium et al., 2012) and the shotgun metagenomic datasets were retrieved using the MicrobeDS R package and curatedMetagenomics R package (Pasolli et al., 2017), respectively. All data was loaded, processed and visualized in the R-environment using Phyloseq to evaluate the prevalence and relative abundance of Dolosigranulum.

For the exploration of Dolosigranulum presence and relative abundance in various environmental, plant, and animal samples, the following version of the Earth Microbiome Project data was downloaded (Gilbert et al., 2014): EMP release 1, subset of 10.000 samples, rarefied to 10.000 reads per sample and classified with release 123 of the SILVA 16 S reference database. Relative abundance data and taxon metadata (e.g., taxonomic classification) were downloaded as a biom file, while sample metadata (e.g., sampling 
location) was downloaded as a tsv file. The relative abundance data and taxon metadata were extracted from the biom file using the biom python package (McDonald et al., 2012). Abundance data, taxon metadata and sample metadata were then integrated and visualized using tidyamplicons version 0.2.0 (github.com/swittouck/tidyamplicons).

\section{Phylogenomic and pangenome analyses}

For the Lactobacillales dataset, one reference genome per species of Carnobacteriaceae and one reference genome per genus of non-Carnobacteriaceae was selected using the GTDB (Parks et al., 2020). Selected genomes were then downloaded from GenBank and genes were predicted with Prodigal v2.6.3 (Hyatt et al., 2010). The pangenome was inferred with Progenomics version 13b9be1 (Wittouck et al., 2019) and genes present in more than 95\% of genomes were considered core. A concatenated alignment of the amino acid sequences of the core genes was then built and trimal v1.4.rev15 (Capella-Gutiérrez et al., 2009) was used to remove columns where more than $5 \%$ of the genomes had a gap. Finally, a maximum likelihood phylogenetic tree was inferred on the trimmed alignment with IQTREE v1.6.11 (Hoang et al., 2018; Kalyaanamoorthy et al., 2017; Nguyen et al., 2015), using the $\mathrm{LG}+\mathrm{F}+\mathrm{I}+\mathrm{G} 4$ model for substitution rates, amino acid frequencies and among-site heterogeneity. The tree was visualized with ggtree v2.0.2 (Yu et al., 2017). Basic file parsing and data exploration and visualization were performed with R v3.6.3 (R Core Team, 2020) and the packages tidyverse v1.3.0 (Wickham et al., 2019), tidygenomes v0.1.3 (Wittouck, 2020b), ggtree v2.0.2 (Yu et al., 2017) and phangorn v2.5.5 (Schliep et al., 2017).

For the D. pigrum dataset, the same pipeline was used, with the following changes. All genomes annotated by the GTDB as D. pigrum were selected (including D. pigrum AMBR11), and MAGs reconstructed by Nayfach et al. (2020) identified as D. pigrum were added. The occurrence of all genes in these genomes and the completeness values of the genomes were jointly estimated by a log likelihood optimization procedure. Genes were considered core if their occurrence as estimated by this procedure was $99 \%$ or greater. Other changes with respect to the Lactobacillales tree were that the top 1,000 single-copy core genes were selected for tree inference, nucleotide sequences instead of amino acid sequences were aligned and alignment positions with a gap in more than $50 \%$ of the genomes were trimmed. The GTR + F + I + G4 model was used for tree inference.

\section{Isolation and whole genome sequencing of Dolosigranulum pigrum AMBR11 and AMBR12 from the healthy URT}

Nasopharyngeal swabs from healthy volunteers were cultivated in liquid brain heart infusion (BHI) broth (International Medical Products), supplemented with $0.5 \%(\mathrm{v} / \mathrm{v})$ Tween 80 (polysorbate) at $37^{\circ} \mathrm{C}$ to promote growth of Dolosigranulum species. Grown cultures were stored at $-80^{\circ} \mathrm{C}$ in $25 \% \mathrm{v} / \mathrm{v}$ glycerol until further identification. Next, bacterial stocks were cultivated on tryptic soy agar (TSA) supplemented with $5 \%$ sheep blood (bioTRADING). Single colonies were further identified with PCR and Sanger sequencing of the 16S rRNA gene using the 27F (5'-AGAGTTTGATCMTGGCTCAG-3') and 1492R (5'-GG TTACCTTGTTACGACTT-3') primers (Lane, 1991; Turner et al., 1999). Dolosigranulum-positive colonies were stored at $-80^{\circ} \mathrm{C}$ in $25 \% \mathrm{v} / \mathrm{v}$ glycerol and growth medium. Total DNA was extracted for whole genome sequencing using the NucleoSpin 96 Tissue kit (Machery-Nagel) with additional lysis step using $20 \mathrm{mg} / \mathrm{mL}$ lysozyme (Sigma Aldrich) and $100 \mathrm{U} / \mathrm{mL}$ mutanolysin (Sigma Aldrich). Whole genome sequencing was performed with the Nextera XT DNA Sample Preparation kit (Illumina), followed by sequencing with the Illumina MiSeq platform ( $2 \times 300$ cycles) at the Center of Medical Genetics Antwerp (University of Antwerp).

Time course analysis of the antimicrobial activity against S. aureus and determination of lactic acid concentration in supernatant

A time course analysis of the antimicrobial activity against $S$. aureus was performed as described previously (De Keersmaecker et al., 2006) with minor modifications. Briefly, an overnight culture of S. aureus was added to the wells of a microtiterplate in a 100-fold dilution and supplemented with cell-free supernatant (CFS) of the LAB under study (i.e., Lacticaseibacillus and Dolosigranulum species). CFS was obtained by centrifugation of a LAB overnight culture grown for $10 \mathrm{~min}$ at $2000 \mathrm{~g}$ at $4^{\circ} \mathrm{C}$. Afterwards, the CFS was filter sterilized ( $0.20 \mu \mathrm{m}$ cellulose acetate) and the concentration of lactic acid in CFS was measured with the commercially available Roche Yellow line kit (Roche, Basel, Swiss). Hexetidine (0.1\%) was used as positive control. MRS brought to $\mathrm{pH} 4.3$ and $\mathrm{BHI}+0.5 \%$ Tween were used as negative controls for Lacticaseibacillus 
and Dolosigranulum, respectively. S. aureus was grown for $30 \mathrm{~h}$, and the optical density (OD) was measured every 30 min at $595 \mathrm{~nm}$ using a Synergy HTX multi-mode reader (Biotek, Drogenbos, Belgium). Each condition was measured at least in triplicate and the average OD was calculated.

\section{Induction of cytokine gene expression in human Calu-3 cells at mRNA level}

One $\mathrm{mL}$ of the bacterial suspensions at a concentration of $10^{8} \mathrm{CFU} / \mathrm{mL}$ in MEM was added to tissue culture plates containing Calu- 3 cells. Bacteria were incubated for four hours with the cells at $37^{\circ} \mathrm{C}$ with $5 \% \mathrm{CO}_{2}$ and $90 \%$ relative humidity to induce cytokine gene expression. After incubation, cells were rinsed three times with prewarmed PBS. MEM was used as negative control. RNA was extracted using the commercially available RNeasy Mini kit (Qiagen), according to the manufacturer's protocol and stored at $-80^{\circ} \mathrm{C} .1 \mathrm{mg}$ of isolated RNA, quantified with Take3 (Biotek), was used for cDNA synthesis using Readyscript ${ }^{\circledR}$ cDNA synthesis mix (Sigma Aldrich). Expression of the reference genes CYC-1 and ATP5B and the expression of genes for cytokines IL-8, IL-1 $\beta$, and TNF- $\alpha$ was quantified by RT-qPCR on a StepOne Plus Real-Time PCR System. Primers were designed on the basis of published sequences (Moretti et al., 2019) and chemically synthesized by Integrated DNA Technologies (IDT) (key resources table). Each cDNA sample was amplified in duplicate with PowerSYBR® Green PCR Master Mix (Applied Biosystems) in a total volume of $20 \mu \mathrm{L}$ with $0.15 \mu \mathrm{M}$ of each primer, $40 \mathrm{ng}$ of cDNA and nuclease-free water. Data are presented as the ratio of the amount of cytokine mRNA to the amount of reference mRNA. Non-template controls (PCR grade water) were included for each run and could not have a Ct value below 35, which was used as threshold for the cytokine analysis. Minimum Information for Publication of Quantitative Real-Time PCR Experiments (MIQE) guidelines were followed (Bustin et al., 2009).

\section{HEK-Blue ${ }^{\text {TM }}$ hTLR2/6 and hTLR2/1 induction experiments}

The ability of bacterial strains to stimulate the TLR2 dimers of TLR2/1 and TLR2/6 was estimated through the use of the HEK-Blue ${ }^{T M}$ hTLR2-TLR1 and the HEK-Blue ${ }^{T M}$ hTLR2-TLR6 reporter cell line (Invivogen). These cells produce secreted embryonic alkaline phosphatase in response to TLR2-TLR1 or TLR2-TLR6 stimulation. Bacterial strains were brought to a final concentration of $10^{7} \mathrm{CFU} / \mathrm{mL}$ in DMEM and UV-inactivated by a four-time exposure to UV for $15 \mathrm{~min}$. Afterwards, UV-inactivated cells were added to the wells (100 $\mu \mathrm{L} /$ well) in four biological replicates. Pam2CSK4 or Pam3CKS4, for TLR2/6 and TLR2/1 experiments respectively, were resuspended and diluted to a final concentration of $50 \mathrm{ng} / \mathrm{mL}$, and used as a positive control. The cells were incubated with the UV-inactivated strains for $24 \mathrm{~h}$ at $37^{\circ} \mathrm{C}, 5 \% \mathrm{CO}_{2}$. The embryonic alkaline phosphatase secreted by the cells was quantified by adding $50 \mu \mathrm{L}$ of supernatant of each well (in duplicate) to $100 \mu \mathrm{L}$ of substrate solution $(1.5 \mathrm{mg} / \mathrm{mL} \mathrm{pNPP}, 150 \mathrm{mM}$ Tris- $\mathrm{HCl}, 150 \mathrm{mM} \mathrm{NaCl}$ and $7.5 \mathrm{mM}$ $\mathrm{MgCl}_{2}$ at $\mathrm{pH}$ 9.5). After 20 min of incubation shielded from light, absorbance was measured at $405 \mathrm{~nm}$ using a Synergy HTX multi-mode reader.

\section{Galleria mellonella survival assay}

To evaluate the safety and tolerability of D. pigrum AMBR11, the larvae were injected in their last prolegs with $10 \mu \mathrm{L}$ of bacterial solution at different concentrations using a Hamilton syringe (Hamilton Company). Two control groups were used, one injected with PBS $(10 \mu \mathrm{L})$ and one without injections to control for general viability. The larvae were kept on petridishes at $37^{\circ} \mathrm{C}$ and monitored daily for survival.

In vivo effect of D. pigrum AMBR11 in a murine model of IL-4-induced barrier dysfunction Mice ( $n=6$ per condition) were pretreated endonasally with $20 \mu \mathrm{L}$ of D. pigrum AMBR11 in PBS at a concentration of $10^{7} \mathrm{CFU} / \mathrm{mL}, 48 \mathrm{~h}$ and $24 \mathrm{~h}$ prior to IL-4 application. Next, with one-hours interval, mice received three times $50 \mu \mathrm{L}$ of IL-4 (250 ng) or PBS. 24 hours after the last nasal application, $20 \mu \mathrm{L}$ FD4 (50 mg/mL PBS, Sigma-Aldrich) was applied endonasally for the evaluation of mucosal permeability. One hour after FD4 application, mice were sacrificed through intraperitoneal injection of Dolethal (Vétoquinol S.A., Lure, France). Serum and nasal mucosa were collected for further analysis. Levels of FD4 were determined in the serum by a fluorescence reader (FLUOstar Omega).

\section{QUANTIFICATION AND STATISTICAL ANALYSIS}

Statistical details of experiments can be found in the figure legends, including the statistical tests used, exact value of $n$, the definition of center, and dispersion and precision measures. Statistical analysis of microbiome data was performed in R using Fisher's exact test for prevalence and Wilcoxon rank-sum test for relative abundance. In vitro and in vivo experimental data were plotted in GraphPad Prism 7.00 and are 
represented as mean values \pm standard deviation. One-way ANOVA and t test (for parametric data)/Mann Whitney test (for non-parametric data) were used in GraphPad Prism 8.4.3 for respectively multiple and pairwise statistical comparisons. The p value was corrected for multiple comparisons using the Holm-Sidak method in GraphPad Prism. The survival curves for Galleria mellonella were plotted, and statistical analysis was performed via a GraphPad Prism 8.4.3 using the de Log rank (Mantel-Cox) test. Where applicable, this test was preceded by the Bonferroni correction for multiple comparisons. 